\title{
A new capacity for plurisubharmonic functions
}

\author{
by \\ ERIC BEDFORD $\left({ }^{1}\right)$ and B. A. TAYLOR $\left({ }^{2}\right)$ \\ Princeton University, Princeton, NJ, U.S.A: \\ and \\ University of Michigan, Ann Arbor, MI, U.S.A. \\ Institute for Advanced Study, Princeton, NJ, U.S.A.
}

\section{Introduction}

If $\Omega$ is a complex manifold, let $P(\Omega)$ denote the plurisubharmonic functions on $\Omega$. Each $u \in P(\Omega)$ is subharmonic with respect to every operator $\Delta_{a}$ which in local coordinates may be written in the form

$$
\Delta_{a}=\sum a_{i j} \frac{\partial^{2}}{\partial z_{i} \partial \bar{z}_{j}}
$$

where $a=\left(a_{i j}\right)$ is a nonnegative Hermitian matrix. We wish here to exploit the fact that plurisubharmonic functions are simultaneously subharmonic with respect to several Laplacians to obtain some results on their local behavior which are stronger than those known for subharmonic functions. We are motivated by the equation

$$
\left(\operatorname{det}\left[\frac{\partial^{2} u}{\partial z_{i} \partial \bar{z}_{j}}\right]\right)^{1 / n}=\frac{1}{n} \inf \left\{\Delta_{a} u: \operatorname{det}\left[a_{i j}\right]=1\right\}
$$

for $u \in P(\Omega) \cap C^{2}(\Omega)$ (c.f. Gaveau [15]); this quantity, in some sense, estimates the extent to which $u$ lies in the interior of $P(\Omega)$. Because of the geometric nature of the cone $P(\Omega)$, it seems that a "potential theory" which can describe the properties of $P(\Omega)$ must necessarily be nonlinear.

The operator $d d^{c}=2 i \partial \partial^{-}$and its exterior powers $\left(d d^{c}\right)^{j}$ are invariant under holomorphic mappings. It is easily seen that in local coordinates

$$
\left(d d^{c} u\right)^{n}=c_{n} \operatorname{det}\left[\frac{\partial^{2} u}{\partial z_{i} \partial \bar{z}_{j}}\right] d V(z)
$$

(1) Sloan Foundation Fellow

(2) Research supported in part by a grant from the National Science Foundation 
The operators (1.1) and (1.2) are clearly related, but the complex Monge-Ampère operator $\left(d d^{c}\right)^{n}$ seems better suited to the purpose of potential theory. In fact, the Dirichlet problem was shown in [4] to be well posed for $\left(d d^{c}\right)^{n}$ and examples were given to show certain difficulties with (1.1). Here, we will use the operator $\left(d d^{c}\right)^{n}$ to replace the Laplacian and prove, in the category of complex analysis, the analogues of some well known results of classical real potential theory. Since our arguments completely avoid the explicit use of kernels and convolutions, they may in some cases also give new proofs to classical results in the case $n=1$.

As in [4], we may define

$$
\left(d d^{c}\right)^{n}: P(\Omega) \cap L^{\infty}(\Omega, \operatorname{loc}) \rightarrow M_{n, n}(\Omega)
$$

where $M_{n, n}(\Omega)$ denotes the space of $(n, n)$ forms on $\Omega$ with (Borel) measure coefficients, with the usual topology of weak convergence of measures. Our first main result, Theorem 2.1, shows that this is actually an extension of the operator $\left(d d^{\mathrm{c}}\right)^{n}$ on smooth functions. The case $n=2$ was proved differently in [5]. It is known [27] that the operator $\left(d d^{c}\right)^{n}$ cannot be defined as an element of $M_{n, n}(\Omega)$ for all $u \in P(\Omega)$. The "correct" domain of definition for $\left(d d^{c}\right)^{n}$ still seems not to be known.

Several capacities have been introduced in connection with various problems of analytic function theory (see e.g., [1], [10], [22], [25], [26], [28]). And it seems, in contrast to the case of $\mathbf{C}^{\mathbf{1}}$, that there may be a large number of different capacities, each arising naturally from a different problem. For the situation at hand, the natural capacity is

$$
C(K, \Omega)=\sup \left\{\int_{K}\left(d d^{c} v\right)^{n}: v \in P(\Omega), 0<v<1\right\}
$$

where $K \subset \Omega$ is compact. This has the property of decreasing under holomorphic mappings; i.e. if $f: \Omega_{1} \rightarrow \Omega_{2}$ is holomorphic, then

$$
C\left(K, \Omega_{1}\right) \geqslant C\left(f(K), \Omega_{2}\right) .
$$

Our second main result, Theorem 3.5, is that every $u \in P(\Omega)$ is quasicontinuous with respect to this capacity. That is, for each $u \in P(\Omega)$ and each $\varepsilon>0$, there is an open set $\mathscr{O} \subset \Omega$ such that $C(\mathscr{O}, \Omega)<\varepsilon$ and $u$ is continuous on the complement of $\mathcal{O}$. For subharmonic functions, this theorem is due to $H$. Cartan [9].

The usefulness of $\left(d d^{c}\right)^{n}$ in replacing the Laplacian is reflected in the comparison theorems we may obtain using it. For instance, we derive a domination principle, Corollary 4.5 , analogous to the one known in classical potential theory. 
We also consider the capacitary plurisubharmonic function $u_{E}^{*}$, the upper semicontinuous regularization of the envelope

$$
u_{E}=\sup \{v: v \in P(\Omega), v<0, v \leqslant-1 \text { on } E\} .
$$

The extremal function $u_{E}^{*}$ is used to give the connection between the capacity $C(E, \Omega)$ and the pluripolar sets; i.e. sets which are locally contained in sets of the form $\{v=-\infty\}$ for some plurisubharmonic function $v$. With Theorems 2.1 and 3.5 it is possible to give a simpler proof of the characterization given in [2] and [3],

$$
C(K, \Omega)=\int\left(d d^{\mathfrak{c}} u_{K}^{*}\right)^{n}
$$

for $K$ a compact subset of $\Omega$. For (1.4), we assume there is a Stein manifold $M$ with $\Omega=\{\varrho<0\} \Subset M$ for some strictly plurisubharmonic $\varrho$ on $M$. However, since all the interesting aspects of our work are local, we will assume that $\Omega$ is a bounded, strongly pseudoconvex domain in $\mathbf{C}^{n}$. In fact, we may always take $\Omega=\left\{z \in \mathbf{C}^{n}:|z|<R\right\}$. The corresponding statements for Stein manifolds will be obvious, and they are omitted.

We also extend the arguments of [2] and [3] to show in Theorem 6.9 that the sets of outer capacity zero are precisely the pluripolar sets. This then gives another proof of Josefson's theorem [17] on the equivalence of locally and globally pluripolar sets.

We consider also the so-called negligible sets, which are those of the form

$$
\mathcal{N}=\left\{w<w^{*}\right\}
$$

where $w=\sup _{\alpha} u_{\alpha}$ is the upper envelope of an arbitrary family of plurisubharmonic functions locally bounded above, and $w^{*}(z)=\lim \sup _{\zeta \rightarrow z} w(\zeta)$ is its upper semicontinuous regularization. In [2] and [3] it was shown that $\mathcal{N}$ is pluripolar if $\left\{u_{\alpha}\right\} \subset P(\Omega) \cap C(\Omega)$. Here we extend this result to the general case and show in Theorem 7.1 that the negligible sets are pluripolar. As a consequence, we find that the capacity defined in (1.3) satisfies the hypotheses of a generalized capacity, and so by Choquet's theorem the $\mathscr{K}$-analytic sets are capacitable. Another consequence is that monotone sequences in $P(\Omega)$ are "quasi-uniform" (Theorem 7.2). These results are then applied to some questions of stability in the interior Dirichlet problem for $\left(d d^{c}\right)^{n}$, and to give results on the "thin" points of sets.

\section{The operators $\left(d d^{c}\right)^{k}$ and $\mathscr{L}_{k}$}

Throughout the paper we use the notion of positive differential forms, or positive currents, as presented in Lelong [18]. This notion was first defined and used by Lelong 
in [21]. If $\psi$ is a positive current of bidegree ( $p, p)$, then $\psi$ is a distribution of order zero. Thus, if $\varphi$ is a positive current of bidegree $(1,1)$ with smooth coefficients, then $\varphi \wedge \psi$ is well defined and is also a positive current. However, we need to use an extension of this multiplication of positive currents.

Recall that given $v^{1}, \ldots, v^{k} \in P(\Omega) \cap L^{\infty}(\Omega$, loc) we may define the positive current $d d^{\mathfrak{c}} v^{1} \wedge \ldots \wedge d d^{\mathfrak{c}} v^{k}$ (inductively) as a distribution if we give its action on a test form $\psi$ of bidegree $(n-k, n-k)$ as

$$
\int_{\Omega} d d^{\mathfrak{c}} v^{1} \wedge \ldots \wedge d d^{\mathfrak{c}} v^{k} \wedge \psi=\int_{\Omega} v^{1} d d^{\mathfrak{c}} v^{2} \wedge \ldots \wedge d d^{\mathfrak{c}} v^{k} \wedge d d^{\mathrm{c}} \psi
$$

(see [4]). The main result of this section shows that $\left(d d^{c}\right)^{k}$ is continuous under decreasing limits. Thus, the definition is justified as an extension of the operator on smooth functions, and, therefore, retains the usual algebraic properties of the operator on smooth functions.

THEOREM 2.1. Let $\left\{v_{j}^{1}\right\}, \ldots,\left\{v_{j}^{k}\right\}$ be decreasing sequences of functions in $P(\Omega) \cap$ $L^{\infty}(\Omega, \mathrm{loc})$ and assume that for all $z \in \Omega$,

$$
\lim _{j \rightarrow \infty} v_{j}^{i}=v^{i} \in P(\Omega) \cap L^{\infty}(\Omega, \operatorname{loc}), \quad 1 \leqslant i \leqslant k .
$$

Then

$$
\lim _{j \rightarrow \infty} d d^{\mathfrak{c}} v_{j}^{1} \wedge \ldots \wedge d d^{\mathfrak{c}} v_{j}^{k}=d d^{\mathfrak{c}} v^{1} \wedge \ldots \wedge d d^{\mathfrak{c}} v^{k}
$$

where the limit is in the weak topology on $M_{k, k}(\Omega)$, the currents of bidegree $(k, k)$ on $\Omega$ of order zero; i.e. which are represented by integration (see e.g. [18] or [15]).

An immediate consequence of Theorem 2.1 is

COROLlARY 2.2. The map $\left(v^{1}, \ldots, v^{k}\right) \mapsto d d^{c} v^{1} \wedge \ldots \wedge d d^{c} v^{k}$ is a symmetric, multilinear map of $\left[P(\Omega) \cap L^{\infty}(\Omega, \text { loc) }]^{k}\right.$ into the cone of nonnegative closed currents of bidegree $(k, k)$.

For the proof of Theorem 2.1 and many other results of this paper, it is convenient to introduce the classes

$$
P^{*}=P^{*}(\Omega, K, \varrho, A, B)
$$

where $\Omega$ is a bounded, strongly pseudoconvex subset of $\mathbf{C}^{n}, K$ is a compact subset of $\Omega, \varrho$ is a strictly plurisubharmonic function on $\bar{\Omega}$ which is a defining function for 
$\Omega=\{\varrho<0\}$, and $A, B$ are constants with $A>0$. A function $u \in P(\Omega) \cap L^{\infty}(\Omega$, loc) belongs to $P^{*}$ if

$$
u(z)=A \varrho(z)+B, \quad z \in \Omega \backslash K .
$$

We will usually not display the lengthy list of parameters $\Omega, K, \varrho, A, B$.

The use of the classes $P^{*}$ is easily seen. Note that the assertion of Theorem 2.1 is a local result. Also, if the functions $v_{j}^{i}$ are changed outside a relatively compact open set $\omega \subset \Omega$, the convergence in $\omega$ will not be changed. Now, if the relatively compact open set $\omega$ is specified and if the $v_{j}^{i}$ converge as in the hypotheses of Theorem 2.1 we can always find constants $A, B>0$ and a compact set $K$ so that all the functions

$$
\bar{v}_{j}^{i}(z)=\left\{\begin{array}{l}
\max \left(v_{j}^{i}(z), A \varrho(z)+B\right), \quad z \in K \\
A \varrho(z)+B, \quad z \in \Omega \backslash K
\end{array}\right.
$$

belong to $P^{*}$ and $\tilde{v}_{j}^{i}(z)=v_{j}^{i}(z)$ for $z \in \omega$. Thus, in proving a local convergence result like Theorem 2.1, we can always assume the functions are smooth outside a compact subset of $\Omega$.

Proof of Theorem 2.1. The proof is by induction on $k$. For $k=1$, the assertion is well known [18]. Assuming the result for values up to $k$, we will prove that

$$
\lim _{j \rightarrow \infty} \int_{\Omega} v_{j}^{0} d d^{c} v_{j}^{1} \wedge \ldots \wedge d d^{c} v_{j}^{k} \wedge d d^{c} \psi=\int_{\Omega} v^{0} d d^{\mathfrak{c}} v^{1} \wedge \ldots \wedge d d^{\mathfrak{c}} v^{k} \wedge d d^{\mathfrak{c}} \psi
$$

for all test forms $\psi$ on $\Omega$. By (2.1), this implies $d d^{c} v_{j}^{0} \wedge \ldots \wedge d d^{c} v_{j}^{k}$ converges in $M_{k+1, k+1}(\Omega)$. And, following our remarks concerning $P^{*}$ (see (2.2)), we can assume that $v_{j}^{i} \in P^{*}=P^{*}(\Omega, K, \varrho, A, B)$. By adding a constant to the $v^{\prime}$ s, we may assume that $B=0$.

Now we will prove (2.3) for $(n-k-1, n-k-1)$ forms $\tilde{\psi}$ which have the following properties:

$d d^{\mathrm{c}} \tilde{\psi} \geqslant 0$ is a positive current on $\Omega$,

$\tilde{\psi}$ is smooth in a neighborhood of $\partial \Omega$,

$$
\text { and } \tilde{\psi}=\mathbf{0} \text { on } \partial \Omega \text {. }
$$

This will suffice to complete the proof, for given any compactly supported test form $\psi$, we may choose $\varepsilon$ small enough that

$$
\tilde{\psi}=-\varrho\left(d d^{c} \varrho\right)^{n-k-1}+\varepsilon \psi
$$


will satisfy (2.4). Now (2.3) will hold for $\tilde{\psi}$ and $-\varrho\left(d d^{c} \varrho\right)^{n-k-1}$, and thus it will hold for $\psi=\varepsilon^{-1}\left(\tilde{\psi}+\varrho\left(d d^{c} \varrho\right)^{n-k-1}\right)$, which is what we wanted.

The first step in the proof of $(2.3)$ is the inequality $\leqslant$ which is a simple consequence of the uppersemicontinuity of $v^{0}$. For later reference we will state it in a slightly more general form. No proof is given, since this is a well known property of weakly convergent nonnegative measures.

LEMMA 2.3. Let $v_{j}$ be a sequence of upper semicontinuous functions which either increase or decrease almost everywhere to an upper semicontinuous function $v \in P(\Omega) \cap L^{\infty}(\Omega, \mathrm{loc})$. Let $\mu$ and $\mu_{j}$ be nonnegative measures all supported on a compact set $K \subset \Omega$ with $\mu_{j} \rightarrow \mu$. Then

$$
\lim _{j \rightarrow+\infty} \int v_{j} d \mu_{j} \leqslant \int v d \mu
$$

Notice that the lemma (with $v_{j}=v_{j}^{0}, \mu_{j}=d d^{c} v_{j}^{1} \wedge \ldots \wedge d d^{\mathrm{c}} v_{j}^{k} \wedge d d^{\mathrm{c}} \psi$ ) proves that $\leqslant$ holds in (2.3) with $d d^{\mathrm{c}} \psi$ replaced by any $(n-k, n-k)$ form $\chi$ which is positive on the compact set $K$ for which $v_{j}^{i} \in P^{*}(\Omega, K, \varrho, A, B)$. This is because $\chi$ can be split up into two parts, $\chi=\chi_{1}+\chi_{2}$ by a partition of unity, where $\chi_{1} \geqslant 0$ and support $\chi_{2} \subset \Omega \backslash K$. We get the inequality $\leqslant$ in $(2.3)$ for $\chi_{1}$, by the lemma. The terms involving $\chi_{2}$ all converge because $v_{j}^{i}=\mathrm{A} \varrho$ on the support of $\chi_{2}$.

To prove the other inequality in (2.3), first assume that all the approximants $v_{j}^{i}$ are smooth on $\Omega$. Then

$$
\begin{aligned}
& \int_{\Omega} v^{0} d d^{\mathfrak{c}} v^{1} \wedge \ldots \wedge d d^{\mathfrak{c}} v^{k} \wedge d d^{\mathfrak{c}} \psi=\int_{K}+\int_{\Omega \backslash K} \\
& \quad \leqslant \int_{K} v_{j}^{0} d d^{\mathfrak{c}} v^{1} \wedge d d^{\mathfrak{c}} v^{2} \wedge \ldots \wedge d d^{\mathfrak{c}} v^{k} \wedge d d^{\mathfrak{c}} \psi+\int_{\Omega \backslash K} v^{0} d d^{\mathfrak{c}} v^{1} \wedge \ldots d d^{\mathfrak{c}} v^{k} \wedge d d^{\mathfrak{c}} \psi \\
& \quad=\int_{\Omega} v_{j}^{0} d d^{\mathfrak{c}} v^{1} \wedge \ldots \wedge d d^{c} v^{k} \wedge d d^{c} \psi+\int_{\Omega \backslash K}\left(v^{0}-v_{j}^{0}\right) d d^{\mathfrak{c}} v^{1} \wedge \ldots \wedge d d^{\mathfrak{c}} v^{k} \wedge d d^{\mathfrak{c}} \psi
\end{aligned}
$$

The last integral vanishes because $v^{0}, v_{j}^{0}=A \varrho$ on $\Omega \backslash K$. Using (2.1), the first integral is equal to

$$
\begin{aligned}
& \int_{\Omega} v^{1} d d^{c} v_{j}^{0} \wedge d d^{c} v^{2} \wedge \ldots \wedge d d^{c} v^{k} \wedge d d^{c} \psi \leqslant \int_{\Omega} v_{j}^{1} d d^{c} v_{j}^{0} \wedge d d^{c} v^{2} \wedge \ldots d d^{c} v^{k} \wedge d d^{c} \psi \\
& =\int_{\Omega} v_{j}^{0} d d^{c} v_{j}^{1} \wedge d d^{c} v^{2} \wedge \ldots \wedge d d^{\mathfrak{c}} v^{k} \wedge d d^{c} \psi .
\end{aligned}
$$


Repeating the argument $k-1$ more times gives

$$
\int_{\Omega} v^{0} d d^{\mathrm{c}} v^{1} \wedge \ldots \wedge d d^{\mathrm{c}} v^{k} \wedge d d^{\mathrm{c}} \psi \leqslant \int_{\Omega} v_{j}^{0} d d^{\mathrm{c}} v_{j}^{1} \wedge \ldots \wedge d d^{\mathrm{c}} v_{j}^{k} \wedge d d^{\mathrm{c}} \psi
$$

Letting $j \rightarrow \infty$ in (2.5) gives the other inequality in (2.3).

If the $v_{j}^{i}$ are not smooth, then note first that it follows from the case just proved that

$$
\lim _{\delta \rightarrow 0} \int_{\Omega}\left(v_{j}^{0}\right)_{\delta} d d^{\mathrm{c}}\left(v_{j}^{1}\right)_{\delta} \wedge \ldots \wedge d d^{\mathrm{c}}\left(v_{j}^{k}\right)_{\delta} \wedge d d^{\mathrm{c}} \psi=\int_{\Omega} v_{j}^{0} d d^{\mathrm{c}} v_{j}^{1} \wedge \ldots \wedge d d^{\mathfrak{c}} v_{j}^{k} \wedge d d^{\mathrm{c}} \psi
$$

where $(v)_{\delta}=v * \psi_{\delta}$ denotes a usual regularization of the plurisubharmonic function $v$ (see [18]). Thus, by a diagonalization process, we can choose $\delta_{j} \rightarrow 0$ so that $\tilde{v}_{j}^{i}=\left(v_{j}^{i}\right) \delta_{j}$ decreases to $v^{i}$ and for all test forms $\psi$

$$
\lim _{j \rightarrow \infty} \int_{\Omega} v_{j}^{0} d d^{c} v_{j}^{1} \wedge \ldots \wedge d d^{\mathrm{c}} v_{j}^{k} \wedge d d^{\mathrm{c}} \psi=\lim _{j \rightarrow \infty} \int_{\Omega}\left(\tilde{v}_{j}^{0}\right) d d^{\mathrm{c}} \tilde{v}_{j}^{1} \wedge \ldots \wedge d d^{\mathrm{c}} \tilde{v}_{j}^{k} \wedge d d^{\mathrm{c}} \psi
$$

By the case just proved, the last limit equals $\int v^{0} d d^{c} v^{1} \wedge \ldots \wedge d d^{c} v^{k} \wedge d d^{c} \psi$. This completes the proof.

The operators

given by

$$
\mathscr{L}_{k}:\left[P(\Omega) \cap L^{\infty}(\Omega, \mathrm{loc})\right]^{k+1} \rightarrow M_{k, k}(\Omega)
$$

$$
\mathscr{L}_{k}\left(v^{0}, v^{1}, \ldots, v^{k}\right)=v^{0} d d^{c} v^{1} \wedge \ldots \wedge d d^{c} v^{k}
$$

which appeared in the proof of Theorem 2.1 are equally as important as $\left(d d^{\mathfrak{c}}\right)^{k}$. Of course, from (2.1), convergence of the currents $d d^{\mathrm{c}} v_{j}^{0} \wedge \ldots \wedge d d^{\mathfrak{c}} v_{j}^{k}$ is equivalent to the convergence of $\mathscr{L}_{k}\left(v_{j}^{0}, \ldots, v_{j}^{k}\right)$ on test forms of the type $d d^{c} \psi$. However, the $\mathscr{L}_{k}$ 's themselves also converge under decreasing limits and this was, essentially, already shown in the proof of Theorem 2.1 .

THEOREM 2.4. Let $\Omega \subset \mathbf{C}^{n}$, and let $\left\{v_{j}^{0}\right\}, \ldots,\left\{v_{j}^{k}\right\}$ be decreasing sequences of functions in $P(\Omega) \cap L^{\infty}(\Omega$, loc $)$ such that

$$
\lim _{j \rightarrow \infty} v_{j}^{i}=v^{i} \in P(\Omega) \cap L^{\infty}(\Omega, \operatorname{loc})
$$

Then

$$
\lim _{j \rightarrow \infty} \mathscr{L}_{k}\left(v_{j}^{0}, \ldots, v_{j}^{k}\right)=\mathscr{L}_{k}\left(v^{0}, \ldots, v^{k}\right),
$$

where the convergence is as currents of order 0 in $M_{k, k}(\Omega)$. 
The main part of the proof of Theorem 2.4 is an observation we will use again later, so it is stated separately here. It is convenient to use the notation $\mu(\varphi)$ for the pairing of a current $\mu$ with a test form $\varphi$ in this lemma.

LEMMA 2.5. Let $\mu, v$ be currents of bidegree $(k, k)$ on $\Omega \subset \mathbf{C}^{n}$ such that

(i) $\mu-v$ is a positive current on $\Omega$

(ii) $\mu=v$ outside a compact subset $K$ of $\Omega$; and

(iii) $\mu\left(d d^{\mathrm{c}} \psi\right)=\nu\left(d d^{\mathrm{c}} \psi\right)$ for all smooth $(n-k-1, n-k-1)$

test forms $\psi$ on $\Omega$.

Then $\mu=v$.

Remark. Note that (iii) implies that $n>k$. The lemma asserts there are no closed nonnegative compactly supported $(k, k)$ forms, if $k<n$.

Proof. Let $\beta_{p}=\beta^{p} / p$ ! where $\beta=\frac{1}{2} i \Sigma d z_{j} \wedge d \bar{z}_{j}$ is the Kähler form on $\mathbf{C}^{n}$. Since $\mu-v \geqslant 0$ and $\mu-v=0$ outside of $K$, we only have to prove that $(\mu-v)\left(\beta_{n-k}\right)=0$ ([18], Theorem 2, p. 69). Let $\chi \geqslant 0, \chi \in C_{0}^{\infty}(\Omega)$ be such that $\chi=1$ on a neighborhood of $K$. Let $u$ be a smooth $(n-k-1, n-k-1)$ form with $d d^{c} u=\beta_{n-k}$. Then

$$
\begin{aligned}
(\mu-v)\left(\beta_{n-k}\right) & =(\mu-v)\left(\chi \beta_{n-k}\right)=(\mu-v)\left(\chi d d^{\mathfrak{c}} u\right) \\
& =(\mu-v)\left(d d^{\mathfrak{c}}(\chi u)-d \chi \wedge d^{\mathfrak{c}} u-d u \wedge d^{\mathfrak{c}} \chi-u d d^{\mathfrak{c}} \chi\right) .
\end{aligned}
$$

Now $(\mu-v)\left(d d^{c}(\chi u)\right)=0$ by hypothesis (iii). All the other terms involve derivatives of $\chi$ and therefore are supported in the open set $\Omega \backslash K$ where $\mu-v$ vanishes. Thus, $\mu-\nu=0$, as asserted.

Proof of Theorem 2.4. Since the assertion of the theorem is local, we may assume all the $v_{j}^{i}$ belong to one of the classes $P^{*}$ (see (2.2)). First assume $n>k$, and let

$$
\mu=v^{0} d d^{\mathfrak{c}} v^{1} \wedge \ldots \wedge d d^{\mathfrak{c}} v^{k}=\mathscr{L}_{k}\left(v^{0}, v^{1}, \ldots, v^{k}\right) .
$$

Let $v$ be any weak limit of the currents $\mathscr{L}_{k}\left(v_{j}^{0}, \ldots, v_{j}^{k}\right)$. By the proof of Theorem 2.1 and the $\leqslant$ part of (2.3), or directly from Lemma 2.3 , it follows that $\mu \geqslant v$. Since $v_{j}^{i} \in P^{*}$, we have $\mu=\nu=\mathscr{L}_{k}(A \varrho+B, \ldots, A \varrho+B)$ outside $K \Subset \Omega$. And Theorem 2.1, via (2.1), implies that $\mu=v$ on test forms of the type $d d^{\mathrm{c}} \psi$. Thus, $\mu=v$ by Lemma 2.5. This proves the Theorem when $n>k$.

If $k=n$, we can think of the $v_{j}^{i}$ as functions on $\Omega \times \mathbf{C} \subset \mathrm{C}^{n+1}$. Then the case just 
proved applies, so $\left\{\mathscr{L}_{n}\left(v_{j}^{0}, \ldots, v_{j}^{n}\right)\right\}$ converges as currents on $\Omega \times \mathbf{C}$. Applying this convergence to test forms of the type $\psi_{1}\left(z_{1}, \ldots, z_{n}\right) \psi_{2}\left(z_{n+1}\right) d z_{n+1} \wedge d \bar{z}_{n+1}$, we see that the $\mathscr{L}_{n}\left(v_{j}^{0}, \ldots, v_{j}^{n}\right)$ also converges as currents (measures) on $\Omega \subset \mathbf{C}^{n}$. This completes the proof.

Of course, the form $\mathscr{L}_{k}\left(v^{0}, v^{1}, \ldots, v^{k}\right)$ is symmetric in $\left(v^{1}, \ldots, v^{k}\right)$, but not in $\left(v^{0}, v^{1}, \ldots, v^{k}\right)$. However, another interesting consequence of Lemma 2.5 is the "symmetry in convergence" of $\mathscr{L}_{k}$ in $\left(v^{0}, v^{1}, \ldots, v^{k}\right)$.

THEOREM 2.6. Suppose $\left\{v_{j}^{0}\right\}, \ldots,\left\{v_{j}^{k}\right\}$ converge pointwise to $v^{0}, \ldots, v^{k}$ in $P(\Omega) \cap L^{\infty}(\Omega$, loc $)$ in such a way that

(i) $\mathscr{L}_{k}\left(v_{j}^{0}, v_{j}^{1}, \ldots, v_{j}^{k}\right) \rightarrow \mathscr{L}_{k}\left(v^{0}, v^{1}, \ldots, v^{k}\right)$; and

(ii) $d d^{c} v_{j}^{0} \wedge d d^{c} v_{j}^{2} \wedge \ldots \wedge d d^{c} v_{j}^{k} \rightarrow d d^{c} v^{0} \wedge d d^{c} v^{2} \wedge \ldots \wedge d d^{c} v^{k}$.

(iii) $\left\{v_{j}^{1}\right\}$ is eitheran increasing or decreasing sequence. Then

$$
\mathscr{L}_{k}\left(v_{j}^{1}, v_{j}^{0}, v_{j}^{2}, \ldots, v_{j}^{k}\right) \rightarrow \mathscr{L}_{k}\left(v^{1}, v^{0}, v^{2}, \ldots, v^{k}\right) .
$$

Proof. If $\mu=\mathscr{L}_{k}\left(v^{1}, v^{0}, v^{2}, \ldots, v^{k}\right)$ and $v$ is any weak limit of $\mathscr{L}_{k}\left(v_{j}^{1}, v_{j}^{0}, v_{j}^{2}, \ldots, v_{j}^{k}\right)$ then $\mu \geqslant \nu$ (by Lemma 2.3), $\mu=v$ outside of $K$, (since we can assume $v_{j}^{i} \in P^{*}$ ). Further, on test forms of the form $d d^{\mathfrak{c}} \psi, \mathscr{L}_{k}$ is symmetric in $v^{0}, \ldots, v^{k}$. Thus, Lemma 2.5 guarantees convergence for the $\mathscr{L}_{k}$ no matter the order of the sequences when $n>k$. The case $n=k$ follows exactly as in the proof of Theorem 2.4.

An important corollary of Theorems 2.1, 2.4 is the following.

THEOREM 2.7. If $\Omega \subset \mathbf{C}^{n}$ and $\left\{u_{j}\right\},\left\{w_{j}\right\},\left\{v_{j}^{1}\right\}, \ldots,\left\{v_{j}^{k}\right\}$ are decreasing sequences of functions in $P(\Omega) \cap L^{\infty}(\Omega, l o c)$, and if the corresponding limits $u, w, v^{1}, \ldots, v^{n} \in$ $P(\Omega) \cap L^{\infty}(\Omega$, loc $)$, then

$$
\lim _{j \rightarrow \infty} d u_{j} \wedge d^{\mathfrak{c}} w_{j} \wedge d d^{\mathfrak{c}} v_{j}^{1} \wedge \ldots \wedge d d^{\mathfrak{c}} v_{j}^{k}=d u \wedge d^{\mathfrak{c}} w \wedge d d^{\mathfrak{c}} v^{1} \wedge \ldots \wedge d d^{\mathfrak{c}} v^{n} \text { in } M_{k+1, k+1}(\Omega)
$$

Proof. By polarization in the symmetric forms $d u \wedge d^{\mathrm{c}} w$ and $d d^{\mathrm{c}} v^{1} \wedge \ldots \wedge d d^{\mathrm{c}} v^{k}$, it is no loss of generality to assume that $u_{j}=w_{j}$ and $v_{j}^{i}=v_{j}, 1 \leqslant i \leqslant k$. Also assume $u \geqslant 0$. Then we have the identity

$$
\frac{1}{2} d d^{\mathfrak{c}} u_{j}^{2} \wedge\left(d d^{\mathrm{c}} v_{j}\right)^{k}=u_{j} d d^{\mathrm{c}} u_{j} \wedge\left(d d^{\mathfrak{c}} v_{j}\right)^{k}+d u_{j} \wedge d^{\mathrm{c}} u_{j} \wedge\left(d d^{\mathfrak{c}} v_{j}\right)^{k}
$$

(which also serves as the weak definition of the last term). Since the left hand side and the first term of the right hand side converge as $j \rightarrow+\infty$, by Theorems 2.1 and 2.4 , so 
does the last term. The general case follows by applying this argument to $u_{j}+C$ for a suitable large constant $C$.

Note the following typical, simple algebraic formulas which hold as a consequence of Theorems 2.1, 2.4, 2.7.

COROLlaRY 2.8. If $u, v \in P(\Omega) \cap L^{\infty}(\Omega$, loc $)$ and $\varphi \in C_{0}^{\infty}(\Omega)$, then $\int \varphi\left[\left(d d^{c} u\right)^{n}-\right.$ $\left.\left(d d^{\mathfrak{c}} v\right)^{n}\right]=\int(u-v) d d^{\mathfrak{c}} \varphi \wedge \theta$, where

$$
\theta=\left(d d^{\mathrm{c}} u\right)^{n-1}+\left(d d^{\mathrm{c}} u\right)^{n-2} \wedge d d^{\mathrm{c}} v+\ldots+\left(d d^{\mathrm{c}} v\right)^{n-1} .
$$

Proof. The equation holds for smooth $u$ and $v$ so the result follows from Theorem 2.1 by approximating $u, v$ by decreasing limits of smooth plurisubharmonic functions.

COROLlaRY 2.9. If $u, v, w \in P^{*}=P^{*}(\Omega, K, \varrho, A, B)$, then

$$
\int_{\Omega}(u-v)\left(d d^{\mathrm{c}} w\right)^{n}=-\int_{\Omega} d(u-v) \wedge d^{\mathfrak{c}} w \wedge\left(d d^{\mathrm{c}} w\right)^{n-1}
$$

Proof. We can select $u_{j}, v_{j}, w_{j}$ smooth plurisubharmonic functions in a class $P^{*}$ (with possibly different $K, A, B$ ) which decrease to $u, v, w$ on a neighborhood of $K$. Then convergence follows from Theorem 2.4 for the left side and Theorem 2.7 for the right side.

Finally, for later reference, we record the Chern-Levine-Nirenberg estimates for $\left(d d^{c}\right)^{n}$ on bounded plurisubharmonic functions (c.f. [11] or [4]).

THEOREM 2.10. If $v^{0}, \ldots, v^{k} \in P(\Omega) \cap L^{\infty}(\Omega), \Omega \subset \mathbf{C}^{n}$, and if $K$ is a compact subset of $\Omega$, then there is a constant $C=C(K, \Omega)$ such that
(i) $\left|\int_{K} d d^{c} v^{1} \wedge \ldots \wedge d d^{c} v^{k} \wedge \beta_{n-k}\right| \leqslant C\left\|v^{1}\right\| \ldots\left\|v^{k}\right\|$
(ii) $\left|\int_{K} v^{0} d d^{c} v^{1} \wedge \ldots \wedge d d^{c} v^{k} \wedge \beta_{n-k}\right| \leqslant C\left\|v^{0}\right\| \ldots\left\|v^{k}\right\|$
(iii) $\left|\int_{K} d v^{0} \wedge d^{c} v^{1} \wedge d d^{c} v^{2} \wedge \ldots \wedge d d^{c} v^{k} \wedge \beta_{n-k}\right| \leqslant C\left\|v^{0}\right\| \ldots\left\|v^{k}\right\|$

where $\beta_{n-k}=\beta^{n-k} /(n-k) !, \beta=\frac{1}{2} i \Sigma d z_{j} \wedge d \bar{z}_{j}$ is the Kähler form, and

$$
\left\|v^{i}\right\|=\sup \left\{\left|v^{i}(z)\right|: z \in \Omega\right\} .
$$

is the supremum norm of $v$. 


\section{Quasicontinuity}

In this section we define the inner capacity associated with the operator $\left(d d^{c}\right)^{n}$ and give some of its elementary properties. Then we give a Schwarz inequality for the gradients of plurisubharmonic functions. The main result of the section, Theorem 3.5 , is that a plurisubharmonic function is continuous off of an open set with arbitrarily small capacity. For subharmonic functions, the corresponding result was obtained by $\mathrm{H}$. Cartan [9].

Definition 3.1. Let $\Omega$ be an open set in $\mathbf{C}^{n}$. If $K$ is a compact subset of $\Omega$, then we define

and

$$
C(K, \Omega)=C(K)=\sup \left\{\int_{K}\left(d d^{\mathrm{c}} u\right)^{n}: u \in P(\Omega), 0<u<1\right\}
$$

$$
C(E, \Omega)=\sup \{C(K): K \text { is a compact subset of } E\} .
$$

Note that $C(E)$ is an inner capacity. If $E$ is a Borel set, then

$$
C(E)=\sup \left\{\int_{E}\left(d d^{c} u\right)^{n}: u \in P(\Omega), 0<u<1\right\} .
$$

PROPOSITION 3.2. Let $\Omega \subset \mathbf{C}^{n}$ and $C(E)=C(E, \Omega)$.

(1) If $E_{1} \subset E_{2}$, then $C\left(E_{1}\right) \leqslant C\left(E_{2}\right)$.

(2) If $E \subset \Omega_{1} \subset \Omega_{2}$, then $C\left(E, \Omega_{1}\right) \geqslant C\left(E, \Omega_{2}\right)$.

(3) $C\left(\bigcup_{j=1}^{\infty} E_{j}\right) \leqslant \sum_{j=1}^{\infty} C\left(E_{j}\right)$.

(4) If $E_{1} \subset E_{2} \subset \ldots$ are Borel sets in $\Omega$, then $C\left(\cup E_{j}\right)=\lim _{j \rightarrow \infty} C\left(E_{j}\right)$.

Proof. Assertions (1), (2), (3) are clear. For assertion (4) let $u \in P(\Omega), 0<u<1$ be chosen so that $C(E) \leqslant \int_{E}\left(d d^{\mathrm{c}} u\right)^{n}+\varepsilon$. Since $\lim _{j \rightarrow \infty} C\left(E_{j}\right) \geqslant \lim _{j \rightarrow \infty} \int_{E_{j}}\left(d d^{\mathrm{c}} u\right)^{n}$ we have $C(E) \leqslant \lim _{j} C\left(E_{j}\right)$. The other inequality follows from (1).

Given a positive $(n-1, n-1)$ current $\chi_{0}$ we may consider the pairing

$$
(\alpha, \beta)=-\int \alpha \wedge J^{*} \beta \wedge \chi_{0}
$$

where $\alpha, \beta$ are smooth real 1 -forms and $J^{*}$ is the adjoint of the almost complex structure, acting on 1 -forms. Since this pairing is symmetric it is an inner product so the Schwarz inequality

holds.

$$
|(\alpha, \beta)|^{2} \leqslant(\alpha, \alpha)(\beta, \beta)
$$


PROPOSITION 3.3. Let $u_{1}, u_{2}, v_{1}, v_{2}, w_{1}, \ldots, w_{n-1} \in P(\Omega) \cap L^{\infty}(\Omega$, loc $)$ be given. If $\left\{u_{1} \neq u_{2}\right\} \Subset \Omega, 0 \leqslant \psi \in C_{0}^{\infty}(\Omega), \psi=1$ on $\left\{u_{1} \neq u_{2}\right\}$, then

$$
\begin{aligned}
\left(\int d\left(u_{1}-u_{2}\right) \wedge\right. & \left.d^{\mathrm{c}}\left(v_{1}-v_{2}\right) \wedge \chi\right)^{2} \leqslant\left(\int d\left(u_{1}-u_{2}\right) \wedge d^{\mathcal{c}}\left(u_{1}-u_{2}\right) \wedge \chi\right) \\
& \times\left(\int \psi d\left(v_{1}-v_{2}\right) \wedge d^{\mathrm{c}}\left(v_{1}-v_{2}\right) \wedge \chi\right)
\end{aligned}
$$

where $\chi=d d^{\mathrm{c}} w_{1} \wedge \ldots \wedge d d^{\mathrm{c}} w_{n-1}$.

Proof. If we set $\chi_{0}=\psi \chi$, then with the notation of (3.1), we have

$$
(d u, d v)=\int \psi d u \wedge d^{\mathrm{c}} v \wedge \chi
$$

The integrands in the proposition are defined for $u \in P(\Omega) \cap L^{\infty}(\Omega$, loc $)$ by Theorem 2.7 . The extension to differences of plurisubharmonic functions is achieved by multilinearity. Theorem 2.7 also implies that $d u$ belongs to the Hilbert space obtained by completing the smooth 1 -forms in the norm $|\alpha|^{2}=(\alpha, \alpha)$ induced by the inner product. Thus the estimate is a special case of the Schwarz inequality in this Hilbert space.

The Schwarz inequality is now used to prove that decreasing sequences of plurisubharmonic functions converge "almost" uniformly.

TheOREM 3.4. Let $u_{j}, u \in P^{*}=P^{*}(\Omega, K, \varrho, A, B)$ and suppose $u_{j}$ decreases to $u$ on

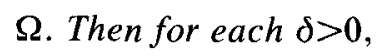

$$
\lim _{j \rightarrow \infty} C\left\{z \in \Omega: u_{j}(z)>u(z)+\delta\right\}=0
$$

(See (2.2) for the definition of $P^{*}$.)

Proof. Without loss of generality, take $\delta=1$. Let $\mathscr{O}_{j}=\left\{z \in \Omega: u_{j}(z)>u(z)+1\right\}$. If $v \in P(\Omega), 0<v<1$, then

$$
\begin{aligned}
\int_{\mathcal{O}_{j}}\left(d d^{\mathfrak{c}} v\right)^{n} & \leqslant \int_{\mathcal{O}_{j}}\left(u_{j}-u\right)\left(d d^{\mathfrak{c}} v\right)^{n} \leqslant \int_{\Omega}\left(u_{j}-u\right)\left(d d^{\mathfrak{c}} v\right)^{n} \\
& =-\int_{\Omega} d\left(u_{j}-u\right) \wedge d^{\mathfrak{c}} v \wedge\left(d d^{\mathfrak{c}} v\right)^{n-1}
\end{aligned}
$$

By Proposition 3.3, the last integral does not exceed

$$
C\left(\int d\left(u_{j}-u\right) \wedge d^{c}\left(u_{j}-u\right) \wedge\left(d d^{c} v\right)^{n-1}\right)^{1 / 2}
$$


where $C=\left(\int_{\omega} d v \wedge d^{\mathrm{c}} v \wedge\left(d d^{\mathrm{c}} v\right)^{n-1}\right)^{1 / 2}$ and $\omega$ is a relatively compact neighborhood of the compact set $K$ from the class $P^{*}=P^{*}(\Omega, K, \varrho, A, B)$. We know the constant $C$ depends only on $\omega$ and $\sup \{|v(z)|: z \in \Omega\}$, by the Chern-Levine-Nirenberg estimate of Proposition 2.10. Another integration by parts then yields

$$
\int_{O_{j}}\left(d d^{\mathrm{c}} v\right)^{n} \leqslant C\left(\int_{\Omega}\left(u_{j}-u\right) \wedge\left(d d^{\mathrm{c}} v\right)^{n-1} \wedge d d^{\mathrm{c}}\left(u_{j}-u\right)\right)^{1 / 2} .
$$

Thus, the power of $d d^{c} v$ has been reduced to $n-1$ in the term on the right hand side of (3.2).

We would like to repeat this argument $n-1$ more times, but there is one problem. Namely, when we integrate by parts to obtain

$$
\int_{\Omega}\left(u_{j}-u\right)\left(d d^{\mathfrak{c}} v\right)^{n-1} \wedge d d^{\mathfrak{c}}\left(u_{j}-u\right)=-\int d\left(u_{j}-u\right) \wedge d^{c} v \wedge\left(d d^{c} v\right)^{n-2} \wedge d d^{c}\left(u_{j}-u\right) .
$$

The Schwarz inequality cannot be applied directly to the right hand side because the $(n-1, n-1)$ current $\left(d d^{c} v\right)^{n-2} \wedge d d^{c}\left(u_{j}-u\right)$ need not be positive. However, it suffices to estimate the integral obtained by replacing $d d^{c}\left(u_{j}-u\right)$ by $d d^{c}\left(u_{j}+u\right)$. This term can then be estimated by the Schwarz inequality and the Chern-Levine-Nirenberg estimate, exactly as before.

Continuing in this manner, we finally obtain that $\int_{\delta_{j}}\left(d d^{c} v\right)^{n}$ is dominated by a finite number $\left(2^{n}\right)$ of terms of the form

$$
C\left(\int\left(u_{j}-u\right) d d^{c} w^{1} \wedge \ldots \wedge d d^{c} w^{n}\right)^{1 / 2^{n-1}}
$$

where each of the functions $w^{1}, \ldots, w^{n}$ is either equal to $u_{j}$ or $u$. The constant $C$ depends on $\sup \left\{\left|u_{j}(z)\right|: z \in \Omega, j=1,2, \ldots\right\}$, which is finite since $u_{j}$ decreases to $u$ in $P^{*}$. According to Theorem 2.4, each integral of the form (3.3) converges to 0 as $j \rightarrow+\infty$. Thus,

$$
\lim _{j \rightarrow \infty}\left[\sup \left\{\int_{O_{j}}\left(d d^{\mathfrak{c}} v\right)^{n}: v \in P(\Omega), 0<v<1\right\}\right]=0,
$$

which completes the proof.

THEOREM 3.5. Let $\Omega$ be a bounded open set in $\mathrm{C}^{n}$ and $u \in P(\Omega)$. Then for each $\varepsilon>0$, there is an open subset $\mathcal{O}$ of $\Omega$ such that $C(\mathcal{O}, \Omega)<\varepsilon$ and $u$ is continuous on $\Omega \backslash \mathcal{O}$. 
To minimize technical problems, we give the proof here only for

$$
u \in P(\Omega) \cap L^{\infty}(\Omega, \text { loc }) .
$$

The full result will be immediate after we have shown that

$$
\lim _{j \rightarrow \infty} C\{z \in \Omega: u(z)<-j\}=0
$$

in Section 6. Only the bounded case of Theorem 3.5 is used in the arguments leading to (3.4).

Proof of Theorem 3.5 for $P(\Omega) \cap L^{\infty}(\Omega$, loc). Because of (2) and (3) of Proposition 3.2 , we can assume that $\Omega$ is strictly pseudoconvex, or even a small ball, and $u$ is plurisubharmonic and bounded on a neighborhood of $\bar{\Omega}$. Let $u_{j}$ be a sequence of smooth plurisubharmonic functions which decrease to $u$ on a neighborhood of $\bar{\Omega}$. By shrinking $\Omega$ and replacing $u_{j}, u$ by $\max \left(u_{j}, A \varrho+B\right)$, we see that it is also no loss of generality to assume that

$$
u_{j}, u \in P^{*}=P^{*}(\Omega, K, \varrho, A, B) .
$$

Then by Theorem 3.4, there exists an integer $j_{l}$ and an open set

$$
\mathscr{O}_{l}^{\prime}=\left\{u_{j_{l}}>u+\frac{1}{l}\right\} \subset \Omega
$$

such that $C\left(\mathcal{O}_{l}^{\prime}, \Omega\right)<2^{-l}$. If $G_{k}=\mathrm{U}_{l>k} \mathcal{O}_{l}^{\prime}$, then the functions $u_{j}$ decrease to $u$ uniformly on $\Omega \backslash G_{k}$. Hence, $u$ is continuous on $\Omega \backslash G_{k}$. But, by (3) of Proposition 3.2, $C\left(G_{k}, \Omega\right) \leqslant \Sigma_{l>k} C\left(\mathcal{O}_{l}^{\prime}, \Omega\right)<2^{-k}$, which completes the proof.

\section{Comparison theorems}

Using the quasicontinuity established in Theorem 3.5, we derive some useful extensions of the results of Section 3 of [4].

THEOREM 4.1. Let $\Omega$ be a bounded open set in $\mathbf{C}^{n}$. Let $u, v \in P(\Omega) \cap L^{\infty}(\Omega)$ and suppose that $\liminf _{\zeta \rightarrow \partial \Omega} u(\xi)-v(\zeta) \geqslant 0$ (i.e. $u \geqslant v$ on $\partial \Omega$ ). Then

$$
\int_{\{u<v\}}\left(d d^{c} v\right)^{n} \leqslant \int_{\{u<v\}}\left(d d^{c} u\right)^{n} .
$$


Proof. First, we can assume that $\liminf _{\zeta \rightarrow a \Omega} u(\zeta)-v(\zeta) \geqslant 2 \delta>0$. Otherwise, replace $u$ by $u+2 \delta$ and then let $\delta \rightarrow 0$. Thus, there is a relatively compact open set $\omega \subset \Omega$ such that $u(z) \geqslant v(z)+\delta$ for $z \in \Omega \backslash \omega$. We can therefore choose smooth plurisubharmonic functions $u_{k}, v_{j}$ which decrease to $u, v$ on a neighborhood of $\bar{\omega}$ and which satisfy $u_{k}(z) \geqslant v_{j}(z)$ for all $z \in \partial \omega$.

By the comparison theorem for smooth plurisubharmonic functions ([4], Proposition 3.1)

$$
\int_{\left\{u_{k}<v_{j}\right\}}\left(d d^{\mathrm{c}} v_{j}\right)^{n} \leqslant \int_{\left\{u_{k}<v_{j}\right\}}\left(d d^{c} u_{k}\right)^{n} .
$$

Let $\varepsilon>0$, and let $G=G_{\varepsilon}$ be an open subset of $\Omega$ with $C(G)<\varepsilon$ such that $u, v$ are continuous on $F=\Omega \backslash G$. Thus, we can write $v=\varphi+\psi$ where $\varphi$ is continuous on $\bar{\Omega}$ and $\psi=0$ outside of $G$. If $U$ is the open set $U=\left\{u_{k}<\varphi\right\}$, then since $\left(d d^{c} v_{j}\right)^{n} \rightarrow\left(d d^{c} v\right)^{n}$ weakly on $\Omega$, we have

$$
\int_{\mathscr{U}}\left(d d^{\mathfrak{c}} v\right)^{n} \leqslant \lim _{j \rightarrow \infty} \int_{\mathscr{U}}\left(d d^{\mathfrak{c}} v_{j}\right)^{n}
$$

But, $\mathscr{U} \subset\left\{u_{k}<v\right\} \cup G$, so

$$
\int_{\mathscr{U}}\left(d d^{\mathfrak{c}} v\right)^{n} \geqslant \int_{\left\{u_{k}<v\right\}}\left(d d^{\mathfrak{c}} v\right)^{n}-\int_{G}\left(d d^{\mathfrak{c}} v\right)^{n}
$$

and

$$
\begin{aligned}
\int_{\mathscr{U}}\left(d d^{\mathfrak{c}} v_{j}\right)^{n} & \leqslant \int_{\left\{u_{k}<v\right\}}\left(d d^{\mathfrak{c}} v_{j}\right)^{n}+\int_{G}\left(d d^{\mathfrak{c}} v_{j}\right)^{n} \\
& \leqslant \int_{\left\{u_{k}<v_{j}\right\}}\left(d d^{\mathfrak{c}} v_{j}\right)^{n}+\int_{G}\left(d d^{\mathfrak{c}} v_{j}\right)^{n} .
\end{aligned}
$$

Thus

$$
\int_{\left\{u_{k}<v\right\}}\left(d d^{c} v\right)^{n} \leqslant \lim _{j \rightarrow \infty} \int_{\left\{u_{k}<v_{j}\right\}}\left(d d^{c} v_{j}\right)^{n}+2\left(M^{n} C(G, \Omega)\right)
$$

where $M=2 \sup \left\{\left|v_{j}(z)\right|: z \in \bar{\omega}, j=1,2, \ldots\right\}$. So if $j \rightarrow+\infty$ in (4.1), we obtain, since $\left\{u_{k}<v_{j}\right\} \searrow\left\{u_{k} \leqslant v\right\}$

$$
\int_{\left\{u_{k}<v\right\}}\left(d d^{\mathfrak{c}} v\right)^{n} \leqslant \int_{\left\{u_{k} \leqslant v\right\}}\left(d d^{c} u_{k}\right)^{n}+2 M^{n} \varepsilon
$$


Now let $k \rightarrow \infty$ in (4.2). The left hand side has limit

$$
\int_{\{u<v\}}\left(d d^{\mathrm{c}} v\right)^{n}
$$

On the set $F$, the functions $u, v$ are continuous, so since $u_{k} \searrow u$,

$$
\int_{\{u \leqslant v\} \cap F}\left(d d^{c} u\right)^{n} \geqslant \lim _{k \rightarrow \infty} \int_{\left\{u_{k} \leqslant v\right\} \cap F}\left(d d^{c} u_{k}\right)^{n} .
$$

Thus

$$
\begin{aligned}
\int_{\{u \geqslant v\}}\left(d d^{\mathfrak{c}} u\right)^{n} & \geqslant \lim _{k \rightarrow \infty} \int_{\{u \leqslant v\} \cap F}\left(d d^{\mathcal{c}^{c}} u_{k}\right)^{n} \\
& \geqslant \lim _{k \rightarrow+\infty} \int_{\left\{u_{k} \leqslant v\right\}}\left(d d^{\mathrm{c}} u_{k}\right)^{n}-\int_{G}\left(d d^{\mathrm{c}} u_{k}\right)^{n} .
\end{aligned}
$$

Consequently, if $M \geqslant 2 \sup \left\{\left|u_{k}(z)\right|: z \in \bar{\omega}, k=1,2, \ldots\right\}$ then we have proved

$$
\int_{\{u<v\}}\left(d d^{\mathfrak{c}} v\right)^{n} \leqslant \int_{\{u \leqslant v\}}\left(d d^{\mathfrak{c}} v\right)^{n}+3 M^{n} \varepsilon .
$$

Since $\varepsilon>0$ is arbitrary, we therefore have

$$
\int_{\{u<v\}}\left(d d^{\mathfrak{c} v}\right)^{n} \leqslant \int_{\{u \leqslant v\}}\left(d d^{c} v\right)^{n} .
$$

Now, in (4.4), replace $u$ by $u+\eta$. The Borel sets $\{u+\eta<v\}$ increase to $\{u<v\}$ as $\eta$ decreases to zero, and the Borel sets $\{u+\eta \leqslant v\}$ increase to $\{u<v\}$. Since $\left[d d^{c}(u+\eta)\right]^{n}=\left(d d^{c} u\right)^{n}$, the theorem then follows from (4.4).

THEOREM 4.2. Let $\Omega$ be a bounded open set in $\mathrm{C}^{n}$ and $u, v \in P(\Omega) \cap L^{\infty}(\Omega)$ be such that $\liminf _{\zeta \rightarrow O \Omega} u(\zeta)-v(\zeta) \geqslant \delta>0$. Then

$$
\int_{\{u \leqslant v\}}\left(d d^{\mathfrak{c} v}\right)^{n} \leqslant \int_{\{u \leqslant v\}}\left(d d^{\mathfrak{c}} v\right)^{n} .
$$

Proof. Let $u_{\varepsilon}=u-\varepsilon$, and $S_{\varepsilon}=\left\{u_{\varepsilon}<v\right\}$. Clearly, $S_{\varepsilon}$ decreases to $\{u \leqslant v\}$ as $\varepsilon$ decreases to zero. By hypothesis, $S_{\varepsilon} \subset \omega \Subset \Omega$ if $\varepsilon$ is sufficiently small. Then, by Theorem 4.1,

$$
\int_{S_{\varepsilon}}\left\{d d^{c} v\right\}^{n} \leqslant \int_{S_{\varepsilon}}\left\{d d^{c} u\right\}^{n}
$$


Letting $\varepsilon \rightarrow 0$, we obtain the desired inequality.

COROLLARY 4.3. Let $\Omega$ be a bounded open set in $\mathrm{C}^{n}$. Let $u, v \in P(\Omega) \cap L^{\infty}(\Omega)$ satisfy

(i) $\lim _{\zeta \rightarrow \partial \Omega} u(\zeta)=\lim _{\zeta \rightarrow \partial \Omega} v(\zeta)=0$,

(ii) $u \leqslant v$ in $\Omega$.

Then

$$
\int_{\Omega}\left(d d^{\mathfrak{c}} v\right)^{n} \leqslant \int_{\Omega}\left(d d^{\mathfrak{c}} u\right)^{n}
$$

Proof. By Theorem 4.1,

$$
\int_{\Omega}\left(d d^{c} v\right)^{n} \leqslant \int_{\Omega}\left[d d^{c}(1+\varepsilon) u\right]^{n}=(1+\varepsilon)^{n} \int_{\Omega}\left(d d^{c} u\right)^{n}
$$

for $\varepsilon>0$, which gives the result.

COROLLARY 4.4. Let $\Omega$ be a bounded open set. If $u, v \in P(\Omega) \cap L^{\infty}(\Omega)$, $\lim \sup _{\zeta \rightarrow \Omega \Omega}|u(\zeta)-v(\zeta)|=0$, and $\left(d d^{\mathrm{c}} u\right)^{n}=\left(d d^{\mathrm{c}} v\right)^{n}$ in $\Omega$, then $u \equiv v$ in $\Omega$.

Proof. It suffices to prove $u \geqslant v$. Let $\psi<0$ be a smooth strongly plurisubharmonic function on $\bar{\Omega}$. If $\{u<v\}$ is not empty, then $S=\{u<v+\varepsilon \psi\}$ is not empty for some $\varepsilon>0$. Further, since $u$ and $v+\varepsilon \psi$ are subharmonic, $S$ has positive Lebesgue measure. By Theorem 4.1,

$$
\int_{S}\left(d d^{c} u\right)^{n} \geqslant \int_{S}\left[d d^{c}(v+\varepsilon \psi)\right]^{n} \geqslant \int_{S}\left(d d^{c} v\right)^{n}+\varepsilon^{n} \int_{S}\left(d d^{c} \psi\right)^{n}
$$

which is a contradiction since the last integral over $S$ is strictly positive.

COROLlaRY 4.5 (domination principle). Let $\Omega$ be a bounded open set and $u, v \in P(\Omega) \cap L^{\infty}(\Omega)$ such that

(i) $\lim \sup _{\zeta \rightarrow \partial \Omega}|u(\zeta)-v(\zeta)|=0$;

and

$$
\text { (ii) } \int_{\{u<v\}}\left(d d^{c} u\right)^{n}=0
$$

Then $u \geqslant v$ in $\Omega$. 
Proof. If we replace $v$ by $v-\varepsilon+\delta|z|^{2}=\tilde{v}$ where $\varepsilon, \delta$ are chosen so that $\tilde{v}<v$ on $\bar{\Omega}$, then

$$
0<\int_{\{u<\bar{v}\}}\left(d d^{c} \tilde{v}\right)^{n} \leqslant \int_{\{u<\bar{v}\}}\left(d d^{c} u\right)^{n} \leqslant \int_{\{u<v\}}\left(d d^{c} u\right)^{n}=0
$$

which is a contradiction unless $\{u<v\}$ is empty.

\section{Negligible sets}

Let $\left\{u_{\alpha}\right\}$ be a family of plurisubharmonic functions on $\Omega$ which is locally bounded from above. Then the function

$$
u(z)=\sup _{\alpha} u_{\alpha}(z)
$$

need not be plurisubharmonic, but its upper semicontinuous regularization

$$
u^{*}(z)=\lim \sup _{\zeta \rightarrow z} u(\zeta) \geqslant u(z)
$$

is plurisubharmonic. A set of the form

$$
N=\left\{z \in \Omega: u(z)<u^{*}(z)\right\}
$$

is called negligible. In this section we will prove that negligible sets have inner capacity zero. Estimates in terms of other capacities have been given in [19], [20], and [23].

PROPOSITION 5.1. If $\Omega$ is a bounded open set in $\mathbf{C}^{n}$, and if $N \subset \Omega$ is negligible, then $C(N, \Omega)=0$.

Remark. After appropriate definitions are given in Section 6, it will be clear from (5.6) that $N$ also has outer capacity zero.

The proof of Proposition 5.1 proceeds by induction on the dimension $n$.

We shall need some further properties of the operator $\left(d d^{c}\right)^{n}$ and the capacity $C(E, \Omega)$. For convenience, we list them in the following two propositions.

Proposition 5.2. Let $\Omega$ be a strictly pseudoconvex set in $\mathbf{C}^{n}$, and $\left\{u_{j}\right\}$ a sequence in $P(\Omega) \cap L^{\infty}(\Omega$, loc $)$ which increases to $u \in P(\Omega) \cap L^{\infty}(\Omega$, loc) almost everywhere on $\Omega$ (Lebesque measure). Then $\left(d d^{\mathrm{c}} u_{j}\right)^{n} \rightarrow\left(d d^{\mathrm{c}} u\right)^{n}$, weakly as measures on $\Omega$.

To study properties of the capacity, we consider the extremal functions associated to a subset $E$ of $\Omega$, 
and

$$
u_{E}(z)=u_{E}(z, \Omega)=\sup \{v(z): v \in P(\Omega), v \leqslant-1 \text { on } E, v<0 \text { on } \Omega\}
$$

$$
u_{E}^{*}(z)=\lim \sup _{\zeta \rightarrow z} u_{E}(\zeta) .
$$

Then $u_{E}^{*} \in P(\Omega)$ and $-1 \leqslant u_{E}^{*} \leqslant 0$.

The following proposition was proved in [2] and [3]. Here we give a more efficient derivation based on Sections 3 and 4 .

Proposition 5.3. Let $\Omega$ be a strictly pseudoconvex subset of $\mathbf{C}^{n}$. If $K \subset \Omega$ is compact, then

(i) $\left(d d^{c} u_{K}^{*}\right)^{n}=0$ on $\Omega \backslash K$;

(ii) $C(K, \Omega)=\int_{\Omega}\left(d d^{\mathrm{c}} u_{K}^{*}\right)^{n}=\int_{K}\left(d d^{\mathrm{c}} u_{K}^{*}\right)^{n}$;

(iii) if $u_{K}^{*}>-1$ on $K$ then $C(K, \Omega)=0$.

The inductive proofs of these propositions will be:

Step 1. Propositions 5.1, 5.2 in $\mathbf{C}^{n}$ implies Proposition 5.2 in $\mathbf{C}^{n+1}$.

Step 2. Proposition 5.2 in $\mathbf{C}^{n}$ implies Proposition 5.3 in $\mathbf{C}^{n}$.

Step 3. Proposition 5.3 in $\mathbf{C}^{n}$ implies Proposition 5.1 in $\mathbf{C}^{n}$.

In the case $n=1$, Proposition 5.2 is a well-known fact of distribution theory, since $d d^{c}$ is a linear operator which on $\mathbf{C}$ is essentially the Laplacian. Thus, by the inductive argument these results hold in all dimensions.

Proof of. Step 1. We have to show that if $\Omega \subset \mathrm{C}^{n+1}$,

$$
\lim _{j \rightarrow \infty} \int_{\Omega} \varphi\left(d d^{c} u_{j}\right)^{n+1}=\int_{\Omega} \varphi\left(d d^{c} u\right)^{n+1}
$$

for all test functions $\varphi \in C_{0}^{\infty}(\Omega)$. That is,

$$
\lim _{j \rightarrow \infty} \int_{\Omega} u_{j}\left(d d^{\mathrm{c}} u_{j}\right)^{n} \wedge d d^{\mathrm{c}} \varphi=\int_{\Omega} u\left(d d^{\mathrm{c}} u\right)^{n} \wedge d d^{\mathrm{c}} \varphi .
$$

First note that if $v \in P(\Omega) \cap L^{\infty}(\Omega)$ and $\psi(z)=\frac{1}{2} i \chi(z) d z_{n+1} \wedge d \bar{z}_{n+1}, \chi \in C_{0}^{\infty}(\Omega)$, then the "Fubini theorem" holds for Lebesque measure $\lambda$ on $\mathbf{C}$ (see [3]):

$$
\int_{\Omega} v\left(d d^{\mathfrak{c}} v\right)^{n} \wedge \psi=\int_{\mathbf{C}} d \lambda\left(z_{n+1}\right) \int_{\Omega\left(z_{n+1}\right)} v\left(\cdot, z_{n+1}\right)\left(d d^{\mathfrak{c}} v\left(\cdot, z_{n+1}\right)\right)^{n} \chi\left(\cdot, z_{n+1}\right)
$$

where $\Omega\left(z_{n+1}\right)=\left\{z \in \mathbf{C}^{n}:\left(z, z_{n+1}\right) \in \Omega\right\}$, and $f\left(\cdot, z_{n+1}\right)$ denotes the function $z \mapsto$ $f\left(z, z_{n+1}\right)$ on $\Omega\left(z_{n+1}\right)$, where $f$ is a function on $\Omega$. This result is clearly true if $v$ is 
smooth. The general case follows by taking smooth plurisubharmonic functions $v_{j}$ decreasing to $v$. The left hand side of (5.3) converges by Theorem 2.4, and the right hand side by Theorem 2.4 and the bounded convergence theorem. By hypothesis $u_{j}\left(\cdot, z_{n+1}\right)$ converges almost everywhere on $\Omega\left(z_{n+1}\right)$ to $u\left(\cdot, z_{n+1}\right)$ for almost every $z_{n+1}$. Thus (5.2) follows from (5.3) by the bounded convergence theorem if we can prove convergence in $\mathbf{C}^{n}$ :

$$
\lim _{j \rightarrow \infty} \int_{\Omega} \varphi u_{j}\left(d d^{\mathfrak{c}} u_{j}\right)^{n}=\int_{\Omega} \varphi u\left(d d^{\mathfrak{c}} u\right)^{n}
$$

for $u_{j}$ increasing to $u$ almost everywhere in $P\left(\Omega \cap L^{\infty}(\Omega\right.$, loc $)$ for $\Omega \subset C^{n}, \varphi \in C_{0}^{\infty}(\Omega)$.

To prove (5.4), we can clearly assume $\varphi \geqslant 0$. By our inductive hypothesis, $\left(d d^{c} u_{j}\right)^{n} \rightarrow\left(d d^{c} u\right)^{n}$. Thus, by Lemma $2.3, \leqslant$ holds in (5.4).

To prove the other inequality, let $\varepsilon>0$ and choose an open set $G \subset \Omega$ such that $C(G, \Omega)<\varepsilon$ and $u, u_{j}$ are all continuous on $F=\Omega \backslash G$. Let $v=\sup \left\{u_{j}: j=1,2, \ldots\right\}$. Then $v$ is lower semicontinuous on $F$, so there exists a continuous function $g$ on $\Omega$ such that $g \leqslant v$ and $\int_{F} \varphi v\left(d d^{\mathfrak{c}} u\right)^{n} \leqslant \int_{F} \varphi g\left(d d^{\mathrm{c}} u\right)^{n}+\varepsilon$. It is no loss of generality to assume that $g, u, u_{j}$, and $v$ are all bounded between 0 and 1 on the support of $\varphi$. Thus,

$$
\begin{aligned}
\int_{\Omega} \varphi v\left(d d^{c} u\right)^{n} & \leqslant \int_{F} \varphi v\left(d d^{\mathfrak{c}} u\right)^{n}+C(G) \\
& \leqslant \int_{F} \varphi g\left(d d^{\mathfrak{c}} u\right)^{n}+C(G)+\varepsilon \\
& \leqslant \int_{\Omega} \varphi g\left(d d^{\mathfrak{c}} u\right)^{n}+C(G)+\varepsilon \\
& =\lim _{j \rightarrow \infty} \int_{\Omega} \varphi g\left(d d^{\mathfrak{c}} u_{j}\right)^{n}+C(G)+\varepsilon \\
& \leqslant \lim _{j \rightarrow \infty} \int_{F} \varphi g\left(d d^{c} u_{j}\right)^{n}+2 C(G)+\varepsilon
\end{aligned}
$$

But the $u_{j}$ are continuous and increase to $v \geqslant g$ on $F$, so by Dini's theorem, the last term does not exceed

$$
\lim _{j \rightarrow \infty} \int_{F} \varphi u_{j}\left(d d^{c} u_{j}\right)^{n}+2 C(G)+\varepsilon \leqslant \lim _{j \rightarrow \infty} \int_{\Omega} u_{j}\left(d d^{\mathrm{c}} u_{j}\right)^{n}+2 C(G)+\varepsilon .
$$

Since $C(G)<\varepsilon$, we have therefore proved that the left hand side of (5.4) dominates

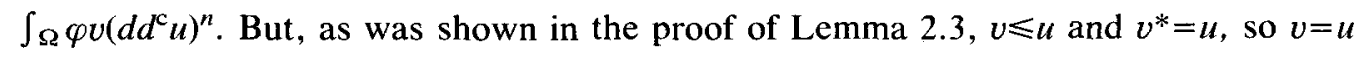


except in the negligible set $N=\left\{v<v^{*}=u\right\}$. By our assumption, $C(N, \Omega)=0$, so $\int_{\Omega} \varphi v\left(d d^{\mathrm{c}} u\right)^{n}=\int_{\Omega} \varphi u\left(d d^{\mathrm{c}} u\right)^{n}$. This completes the proof of Step 1 .

Proof of Step 2. We first prove (i). Note that by Dini's theorem

$$
u_{K}(z)=\sup \{v(z): v \in P(\Omega) \cap C(\bar{\Omega}), v<0, v \leqslant-1 \text { on } K\} .
$$

From (5.5) and Choquet's lemma $\left[18\right.$, p. 26] there is a sequence $u_{1} \leqslant u_{2} \leqslant \ldots$ of functions in $P(\Omega) \cap C(\bar{\Omega})$ with $u_{j} \leqslant-1$ on $K, u_{j}<0$ on $\Omega$, and $u_{K}^{*}=\left(\sup u_{j}\right)^{*}$. If $B$ is a ball in $\Omega \backslash K$, then let $w_{j}$ be the unique, continuous plurisubharmonic function on $B$ with $w_{j}=u_{j}$ on $\partial B \subset \Omega$ and $\left(d d^{\mathrm{c}} w_{j}\right)^{n}=0$ in $B$. Then set

$$
\tilde{u}_{j}=\left\{\begin{array}{lll}
u_{j} & \text { in } & \Omega \backslash B \\
w_{j} & \text { in } B
\end{array}\right.
$$

Then $\tilde{u}_{j} \geqslant u_{j}$ and $\tilde{u}_{1} \leqslant \tilde{u}_{2} \leqslant \ldots$ (See [4], p. 42 for this construction.) Hence, $u_{K}^{*}=\left(\sup \tilde{u}_{j}\right)^{*}$. But, $\left(d d^{c} \tilde{u}_{j}\right)^{n}=0$ on $B$, and $\left(d d^{c} \tilde{u}_{j}\right)^{n} \rightarrow\left(d d^{c} u_{K}^{*}\right)^{n}$, by our inductive assumption that Proposition 5.2 holds. Hence, $\left(d d^{c} u_{K}^{*}\right)^{n}=0$ on $B$. Since $B \subset \Omega \backslash K$ is an arbitrary ball, we have $\left(d d^{c} u_{K}^{*}\right)^{n} \equiv 0$ on $\Omega \backslash K$, so (i) holds.

To prove (ii), let $v \in P(\Omega), \varepsilon<v<1-\varepsilon$. Let $u_{j} \in P(\Omega) \cap C(\bar{\Omega})$ satisfy $u_{j} \leqslant-1$ on $K$, $u_{j}<0$ on $\Omega$, and $\left(\sup u_{j}\right)^{*}=u_{K}^{*}$. We can clearly assume $u_{j} \geqslant A \varrho$ for some $A>0$; otherwise, replace $u_{j}$ by $\max \left(u_{j}, A \varrho\right)$. Thus $\left\{u_{j}<v\right\} \subset\{A \varrho<v\} \Subset \Omega$, and $K \subset\left\{u_{j}<v\right\}$. Therefore, by the comparison Theorem 4.1,

$$
\int_{K}\left(d d^{c} v\right)^{n} \leqslant \int_{\left\{u_{j}<v\right\}}\left(d d^{c} v\right)^{n} \leqslant \int_{\left\{u_{j}<v\right\}}\left(d d^{c} u_{j}\right)^{n}
$$

Since, $\left(d d^{\mathrm{c}} u_{j}\right)^{n} \rightarrow\left(d d^{c} u_{K}^{*}\right)^{n}$, we let $j \rightarrow \infty$ to obtain

$$
\int_{K}\left(d d^{\mathfrak{c}} v\right)^{n} \leqslant \int_{\{A Q<v\}}\left(d d^{\mathfrak{c}} u_{K}^{*}\right)^{n}=\int_{K}\left(d d^{\mathfrak{c}} u_{K}^{*}\right)^{n}
$$

Thus, $C(K, \Omega) \leqslant \int_{K}\left(d d^{c} u_{K}^{*}\right)^{n}$. Since the other inequality is trivial, part (ii) follows.

To prove (iii), we can assume $u_{K}^{*} \geqslant-1+\eta, \eta>0$, on $K$, by (1) and (3) of Proposition 3.2. Then $u=u_{K}^{*} /(1-\eta)+1$ satisfies $u \in P(\Omega), 0 \leqslant u \leqslant 1$, so

$$
C(K) \geqslant \int_{K}\left(d d^{c} u\right)^{n}=\left(\frac{1}{1-\eta}\right)^{n} \int_{K}\left(d d^{c} u_{K}^{*}\right)^{n}=\left(\frac{1}{1-\eta}\right)^{n} C(K),
$$

a contradiction unless $C(K)=0$. This completes the proof of Step 2 . 
Proof of Step 3. By Theorem 3.5 we can choose an open set $G \subset \Omega$ such that $u_{j}, u$ are continuous on $\Omega \backslash G=F$ and $C(G, \Omega)<\varepsilon$. We claim that there exist countably many compact sets $K_{j} \subset F$ such that

$$
N \subset G \cup\left[\cup K_{j}\right]
$$

where $C\left(K_{j}\right)=0$. If this is proved, then by Proposition $3.2, C(N) \leqslant C(G)+\Sigma C\left(K_{j}\right)<\varepsilon$, so $C(N)=0$ and the proposition is proved. Clearly, $N \subset G \cup\left[\left(F \cap\left\{u<u^{*}\right\}\right)\right]$. But, if $K_{\alpha \beta}=\left\{z \in F: u(z) \leqslant \alpha<\beta \leqslant u^{*}(z)\right\}$, then $K_{\alpha \beta} \subset F$ is compact because $u^{*}$ is upper semicontinuous and $u$ is lower semicontinuous on $F$. It is also clear that $F \cap\left\{u<u^{*}\right\}=\mathrm{U} K_{\alpha \beta}$ where the union is over the countably many pairs of rational numbers $(\alpha, \beta)$ with $\alpha<\beta$. Thus, we only have to prove that $C\left(K_{\alpha \beta}\right)=0$. By subtracting a real constant, we may assume that $u_{j}, u<0$ on $\Omega$. Multiplying by another constant, we may set $\alpha=-1$. Thus $u_{K_{\alpha \beta}}^{*} \geqslant u^{*} \geqslant-1=\alpha$ on $K_{\alpha \beta}$. Consequently, $C\left(K_{\alpha \beta}\right)=0$ by Proposition 5.3, and the proof is complete.

\section{Pluripolar sets}

In this section we give the basic connections between pluripolar sets, the extremal functions $u_{E}^{*}$, and the outer capacity $C^{*}$ obtained from $C$. The standard construction of outer capacity is

$$
C^{*}(E)=C^{*}(E, \Omega)=\inf \{C(\mathcal{O}): \mathscr{O} \supset E, \mathscr{O} \text { open in } \Omega\}
$$

Let us remark that if $O \Subset \Omega$ is an open set, and if $K_{1} \subset K_{2} \subset \ldots$ is a sequence of compact sets such that $U K_{j}=\mathcal{O}$, then $\lim _{j \rightarrow \infty} u_{K_{j}}^{*}=u_{C}=u_{C}^{*}$. It follows from Theorem 2.1 and Proposition 5.3 then, that

$$
C(\mathcal{O}, \Omega)=\int\left(d d^{c} u_{\odot}\right)^{n}
$$

for open sets $O \Subset \Omega$.

First, some elementary properties of the outer capacity.

Proposition 6.1. If $\Omega$ is bounded in $\mathbf{C}^{n}$, then

(i) $C^{*}\left(E_{1}\right) \leqslant C^{*}\left(E_{2}\right)$ if $E_{1} \subset E_{2} \subset \Omega$;

(ii) $C^{*}\left(E, \Omega_{1}\right) \geqslant C(E, \Omega)$ if $E \subset \Omega_{1} \subset \Omega$;

(iii) $C^{*}\left(\cup E_{j}, \Omega\right) \leqslant \Sigma_{j} C^{*}\left(E_{j}, \Omega\right)$. 
Proof. These follow immediately from the corresponding properties for $C(E, \Omega)$ (see Proposition 3.2).

Next, some elementary properties of the extremal functions.

Proposition 6.2. If $\Omega$ is bounded in $\mathrm{C}^{n}$, then

(i) $u_{E_{1}}^{*} \geqslant u_{E_{2}}^{*}$ if $E_{1} \subset E_{2} \subset \Omega$;

(ii) $u_{E}^{*}\left(z, \Omega_{1}\right) \geqslant u_{E}^{*}(z, \Omega)$ if $E \subset \Omega_{1} \subset \Omega$;

(iii) if $u_{E_{j}}^{*} \equiv 0$, and $E=\bigcup_{j=1}^{\infty} E_{j}$, then $u_{E}^{*} \equiv 0$.

In addition, if $\Omega$ is strongly pseudoconvex and $E$ is relatively compact in $\Omega$, then

(iv) $u_{E}^{*}(z) \rightarrow 0$ as $z \rightarrow \partial \Omega$.

Proof. Parts (i), (ii) are obvious. So is part (iv), since $0 \geqslant u_{E}^{*} \geqslant A \varrho$ for some $A>0$, where $\Omega=\{\varrho<0\}, \varrho$ a plurisubharmonic defining frunction for $\Omega$. To prove (iii), note the following equivalence which is a direct consequence of Choquet's lemma.

$$
\begin{gathered}
u_{E}^{*} \equiv 0 \text { if and only if there exists a sequence of functions } \\
v_{1} \leqslant v_{2} \leqslant \ldots, \quad v_{j} \in P(\Omega), v_{j}<0 \text { on } \Omega, v_{j} \leqslant-1 \text { on } E \text {, and } \int_{\Omega}\left|v_{j}\right| \leqslant 2^{-j} .
\end{gathered}
$$

Thus, if $v_{j}$ is $\leqslant-1$ on $E_{j}, v_{j}<0$ on $\Omega$, and $\int\left|v_{j}\right| \leqslant \varepsilon 2^{-j}$, then $v=\sum v_{j} \in P(\Omega), v<0, v \leqslant-1$ on $\cup E_{j}$ and $\int|v| \leqslant \varepsilon$. Hence, $u_{E}^{*} \equiv \mathbf{0}$.

We also record another consequence of (6.3).

PROPOSITION 6.3. Let $\Omega$ be bounded in $\mathbf{C}^{n}$. Then $u_{E}^{*} \equiv 0$ if and only if there exists $v \in P(\Omega), v<0$ on $\Omega$ and $E \subset\{z \in \Omega: v(z)=-\infty\}$.

Proof. If $u_{E}^{*} \equiv 0$ and the $v_{j}$ are as in (6.3), then $v=\Sigma v_{j} \in P(\Omega)$ and $E \subset\{z \in \Omega$ : $v(z)=-\infty\}$. Conversely, $E \subset\{v=-\infty\}$, where $v<0, v \in P(\Omega)$ implies $u_{E} \geqslant \sup \{v / j$ : $j=1,2, \ldots\}$, so $u_{E}=0$ on the complement of a set of measure zero. Hence, $u_{E}^{*} \equiv 0$.

Proposition 6.4. Let $\Omega \Subset \mathbf{C}^{n}$ be strongly pseudoconvex. Let $K_{1} \supset K_{2} \supset \ldots$ be a sequence of compact subsets of $\Omega$, and let $K=\cap K_{j}$. Then

(i) $\left(\lim _{j \rightarrow \infty} u_{K_{j}}^{*}\right)^{*}=u_{K}^{*}$,

(ii) $\lim _{j \rightarrow \infty} C\left(K_{j}\right)=\mathrm{C}(K)$,

(iii) $r^{*}(K)=\mathrm{C}(K)$.

Proof. Assertion (i) is well-known and may be found, for instance, in [28], Lemma 1. It is a consequence of Dini's theorem and the lower semicontinuity of the negative of 
the characteristic function of $K$. Assertion (ii) then follows directly from (i) by Propositions 5.2 and 5.3. Assertion (iii) follows from (ii) if we take $K_{j}=\{z \in \Omega$ : distance from $z$ to $K \leqslant 1 / j\}$.

Proposition 6.5. Let $\Omega \Subset \mathrm{C}^{n}$ be strongly pseudoconvex. If $E \Subset \Omega$, then

$$
C^{*}(E)=\int_{\Omega}\left(d d^{c} u_{E}^{*}\right)^{n}
$$

Proof. First, we note from Corollary 4.3 and (6.2) that if $E \subset \mathscr{O} \subseteq \Omega, \mathcal{O}$ an open set, then

$$
\int\left(d d^{c} u_{E}^{*}\right)^{n} \leqslant \int\left(d d^{c} u_{C}^{*}\right)^{n}=C(O)
$$

Thus,

$$
\int\left(d d^{c} u_{E}^{*}\right)^{n} \leqslant C^{*}(E)
$$

For the other inequality, it suffices to show there exist open sets $\mathcal{O}_{1} \supset \mathscr{O}_{2} \supset \ldots$ such that $\Omega \ni \mathcal{O}_{j} \supset E$ and $\left(\lim _{j \rightarrow \infty} u_{\mathscr{\sigma}_{j}}^{*}\right)^{*}=u$. For then, by Proposition 5.2,

$$
C^{*}(E) \leqslant \lim _{j \rightarrow \infty} C\left(\mathcal{O}_{j}\right)=\lim _{j \rightarrow \infty}\left(d d^{c} u_{\delta_{j}}^{*}\right)^{n}=\int\left(d d^{c} u_{E}^{*}\right)^{n}
$$

By Choquet's lemma there exists an increasing sequence $\left\{v_{j}\right\} \subset P(\Omega) \cap L^{\infty}(\Omega)$ such that $v_{j}(z) \rightarrow 0$ as $z \rightarrow \partial \Omega$ and $\lim _{j \rightarrow \infty} v_{j}=u_{E}$ almost everywhere on $\Omega$. If $\mathcal{O}_{j}=\left\{(1+1 / j) v_{j}<-1\right\}$, then $E \subset \mathscr{O}_{j}$ and $v_{j} \leqslant u_{O_{j}} \leqslant u_{E}$. Thus $\lim _{j \rightarrow \infty} u_{\mathscr{O}_{j}}=u_{E}$ a.e., which completes the proof.

Remark. It follows that $u_{E}^{*}=u_{G}^{*}$ where $G=\cap O_{j}$.

COROLLARY 6.6. Let $\Omega \Subset C^{n}$ be strongly pseudoconvex. If $E \subset \Omega$, then $C^{*}(E)=0$ if and only if $u_{E}^{*}=0$.

Proof. If $E \Subset \Omega$, then the assertion is a direct consequence of Proposition 6.4 and the uniqueness result of Corollary 4.4. The general case follows from the countable subadditivity of $C^{*}$ (Proposition 6.1) and the corresponding property for $u_{E}^{*}$ (Proposition 6.2).

Definition 6.7. A set $E$ in $\mathbf{C}^{n}$ is pluripolar if for each $z \in E$ there is an open set $\mathscr{\jmath} z$. and $u \in P(\mathscr{U})$ such that $E \cap \mathscr{U} \subset\{u=-\infty\}$. 
Using the results obtained so far, we can give a simple proof of the following important theorem of Josefson [16]. The argument given here was given in [2] for the case $n=2$, and it works without change in $\mathbf{C}^{n}$, given the relevant properties of $C^{*}$. We include the argument only for the convenience of the reader. Sadullaev [24] has indicated how one may modify the arguments of [2] to give another proof of Josefson's theorem without using the continuity of $\left(d d^{c}\right)^{n}, n \geqslant 2$. As was noted in ([3], Remark 1), Josefson's theorem automatically covers the case where $\mathrm{C}^{n}$ is replaced by an irreducible Stein space. $\left({ }^{l}\right)$

THEOREM 6.8 (Josefson). If $E \subset \mathbf{C}^{n}$ is pluripolar then there exists $u \in P\left(\mathbf{C}^{n}\right)$ with $E \subset\{u=-\infty\}$.

Proof. Since $E$ is pluripolar, we can find sets $E_{j}, \Omega_{j}$ with $E_{j} \subset \Omega_{j} \Subset C^{n}$ such that $\cup_{j=1}^{\infty} E_{j}=E$, each $\Omega_{j}$ is strictly pseudoconvex, and, by Proposition $6.3, u^{*}\left(E_{j}, \Omega_{j}\right) \equiv 0$. Let $i_{1}, i_{2}, \ldots$, be a listing of the positive integers so that each one appears infinitely often. For a sequence $c_{1}<c_{2}<\ldots, \quad c_{j} \rightarrow+\infty$, set $M_{j}=\left\{z \in \mathbf{C}^{n}:|z|<c_{j}\right\}$. We can choose $c_{j}$ large enought that $\Omega_{i_{j}} \Subset M_{j}$ and $|z|-c_{j}<-1$ on $E_{i_{j}}$.

It then follows from Propositions 6.1, 6.3 and Corollary 6.6 and $u^{*}\left(E_{i_{j}}, \Omega_{i_{j}}\right) \equiv 0$, that $u^{*}\left(E_{i_{j}}, M_{j}\right) \equiv 0$. Then, by (6.3), there exists $h_{j} \in P\left(M_{j}\right)$ with $h_{j}<0$ on $M_{j}, h_{j} \leqslant-1$ on $E_{i_{j}}$, and $\int_{M_{j}}\left|h_{j}\right| d V<2^{-j}$. If we set

$$
p_{j}(z)=\left\{\begin{array}{l}
\max \left(h_{j}(z),|z|-c_{j}\right), \quad z \in M_{j} \\
|z|-c_{j}, \quad z \in \mathbf{C}^{n} \backslash M_{j}
\end{array}\right.
$$

then $p_{j} \in P\left(\mathbf{C}^{n}\right)$ and $p_{j} \leqslant-1$ on $E_{i_{j}}$. Further

$$
p(z)=\sum_{j=1}^{\infty} p_{j}(z)
$$

is a plurisubharmonic function on $\mathbf{C}^{n}$ with $p=-\infty$ on $E$ because the sum converges in $L^{1}\left(\mathbf{C}^{n}\right.$, loc $)$, the partial sums are eventually decreasing on each compact subset of $\mathbf{C}^{n}$, and each $E_{j}$ appears infinitely often in the sequence $E_{i_{j}}$. This completes the proof.

We now prove the main result of this section, the characterization of pluripolar sets as sets of outer capacity zero.

THEOREM 6.9. Let $\Omega \Subset \mathrm{C}^{n}$ be strongly pseudoconvex. Then $E \subset \Omega$ is pluripolar if and only if $C^{*}(E)=0$.

(1) Added in proof. A result which generalizes Josefson's theorem was given by the first author in "The operator $\left(d d^{c}\right)^{n}$ on complex spaces", to appear in Séminaire Lelong-Skoda. 
Proof. If $E \subset \Omega$ is pluripolar, then by Theorem 6.8 there exists $u \in P\left(C^{n}\right), u<0$ on $\Omega$ with $E \subset\{u=-\infty\}$. Hence $C^{*}(E)=0$ by Proposition 6.3 and Corollary 6.6. On the other hand, if $C^{*}(E)=0$, then $u_{E}^{*}=0$ by Corollary 6.6 , so $E$ is pluripolar by Proposition 6.3.

We now give the result needed to complete the proof of Theorem 3.5 (the unbounded case).

THEOREM 6.10. If $\Omega$ is open in $\mathbf{C}^{n}$, then for $\omega \Subset \Omega$ and $u \in P(\Omega)$,

$$
\lim _{j \rightarrow \infty} C(\{u<-j\} \cap \omega)=0 .
$$

Proof. We can assume $u<0$ on $\omega$. Since $\bar{\omega}$ can be covered by a finite union of balls in $\Omega$, we may apply Proposition 6.1 and assume that $\Omega$ is strongly pseudoconvex. Then if $\mathcal{O}_{j}=\{u<-j\} \cap \omega$ we have $0 \geqslant u_{\mathcal{O}_{j}} \geqslant \max \{u / j,-1\}$. Thus, $\lim _{j \rightarrow \infty} u_{\mathcal{O}_{j}}=0$ a.e. on $\Omega$ so by (6.2) and Proposition 5.2, $\lim _{j \rightarrow \infty} C\left(\mathcal{O}_{j}, \Omega\right)=0$.

\section{Sequences of plurisubharmonic functions}

In this section we first show that the negligible sets discussed in Section 5 are, in fact, pluripolar. For subharmonic functions, this is due to $\mathrm{H}$. Cartan [8] and is a well known fact of classical potential theory. For plurisubharmonic functions, this settles a question of Lelong ([18], p. 30). Then, Theorem 3.5 is extended to monotone sequences of plurisubharmonic functions; they converge "almost" uniformly. This yields a strengthening of the convergence Theorems $2.1,2.4,2.7$ and 3.2. Finally, we introduce a space $H(\Omega, \operatorname{loc})$ related to the Dirichlet-type norms used in the proof of Theorem 3.4 , and give some of its properties.

THEOREM 7.1. Negligible sets are pluripolar.

Proof. The result is local, so we may consider all the functions as being defined on a bounded strongly pseudoconvex set $\Omega$. The theorem was mostly proved in Section 5 , Proposition 5.1, where we showed that for $\varepsilon>0$, it was possible to find an open set $G$ with $C(G)<\varepsilon$ and countably many compact sets $K_{j}$ with $C\left(K_{j}\right)=0$ such that $N \subset G \cup\left(\cup K_{j}\right)$ (see (5.6)). By Proposition 6.4, $C^{*}(N) \leqslant C^{*}(G)+\Sigma C^{*}\left(K_{j}\right)<\varepsilon$. Therefore, $C^{*}(N)=0$ so $N$ is pluripolar by Theorem 6.9.

THEOREM 7.2. Let $\left\{u_{j}\right\}$ be a monotone sequence of plurisubharmonic functions, either increasing or decreasing, on a bounded open set $\Omega \subset \mathbf{C}^{n}$. Suppose further that $u_{j} \rightarrow u$ almost everywhere on $\Omega$, where $u \in P(\Omega)$. Then for each $\varepsilon>0$, there is an open set 
$G \subset \Omega$ such that $C(G), \Omega)<\varepsilon, u_{j}, u$ are continuous, on $\Omega \backslash G$, and $u_{j}$ converges to $u$ uniformly on compact subsets of $\Omega \backslash G$.

Proof. Let $N=\left\{u \neq \lim _{j \rightarrow \infty} u_{j}\right\}$. By Theorem $1, C^{*}(N, \Omega)=0$. By Theorem 3.5 and the countable subaddivity of $C$, there is an open set $G \supset N$ with $C(G, \Omega)<\varepsilon$ and $u_{j}, u$ continuous on $\Omega \backslash G$. Then $u_{j} \rightarrow F u$ uniformly on compact subsets of $\Omega \backslash G$ by Dini's theorem.

COROLLARY 7.3. Let $\left\{u_{j}\right\}$ be a sequence of plurisubharmonic functions locally bounded above on the bounded open set $\Omega$. Suppose further that

$$
\limsup _{j \rightarrow \infty} u_{j}
$$

is not identically $-\infty$ on any component of $\Omega$. Then there exists $u \in P(\Omega)$ such that $\left\{u \neq \lim \sup u_{j}\right\}$ is pluripolar.

Proof. Set $\tilde{u}_{j}=\sup \left\{u_{k}: k \geqslant j\right\}$. By Theorem 7.1 there exists $v_{j} \in P(\Omega)$ such that $v_{j}=\tilde{u}_{j}$ except on a pluripolar set. The functions $v_{j}$ decrease and

$$
\limsup _{j \rightarrow \infty} u_{j}=\lim _{j \rightarrow \infty} \tilde{u}_{j}=\lim v_{j}
$$

holds except on a pluripolar set. Thus $u=\lim v_{j} \in P(\Omega)$ is the desired function.

Remark. An example of Choquet [13] shows that lim sup cannot be replaced by lim, even if we pass to a subsequence.

THEOREM 7.4. Let $\left\{u_{j}^{i}\right\} \subset P(\Omega) \cap L^{\infty}(\Omega, \operatorname{loc}), 0 \leqslant i \leqslant k(k \leqslant n)$ be $k+1$ sequences that are uniformly bounded on compact subsets of $\Omega$. Suppose there exist $u^{0}, \ldots, u^{k} \in P(\Omega) \cap L^{\infty}(\Omega$, loc $)$ such that $\lim _{j \rightarrow \infty} u_{j}^{i}=u^{i}$ almost everywhere on $\Omega, 0 \leqslant i \leqslant k$. If all but one of the sequences $\left\{u_{j}^{0}\right\}, \ldots,\left\{u_{j}^{k}\right\}$, are monotone, either increasing or decreasing then

(i) $\lim _{j \rightarrow \infty} d d^{c} u_{j}^{1} \wedge \ldots \wedge d d^{c} u_{j}^{k}=d d^{c} u^{1} \wedge \ldots \wedge d d^{c} u^{k}$

(ii) $\lim _{j \rightarrow \infty} u_{j}^{0} d d^{c} u_{j}^{1} \wedge \ldots \wedge d d^{c} u_{j}^{k}=u^{0} d d^{c} u^{1} \wedge \ldots \wedge d d^{c} u^{k}$

(iii) $\lim _{j \rightarrow \infty} d u_{j}^{0} \wedge d^{\mathfrak{c}} u_{j}^{1} \wedge d d^{\mathfrak{c}} u_{j}^{2} \wedge \ldots \wedge d d^{\mathfrak{c}} u_{j}^{k}=d u^{0} \wedge d^{\mathfrak{c}} u^{1} \wedge d d^{\mathfrak{c}} u^{2} \wedge \ldots \wedge d d^{\mathfrak{c}} u^{k}$,

where the limits are in the sense of currents.

We omit the proof, since it parallels exactly the development made in Section 2. The only change is that the monotonicity used in the proof of $\geqslant$ in $(2.3)$ is replaced by 
the almost uniform convergence given by Theorem 7.2. See also ([3], Theorem 1). The induction starts with $k=1$ and $d d^{\mathrm{c}}$ applied on the nonmonotone sequence.

We consider the norm

$$
\|\varphi\|=\sup \left\{\int_{\Omega} d \varphi \wedge d^{c} \varphi \wedge\left(d d^{c} v\right)^{n-1}: v \in P(\Omega), 0<v<1\right\}
$$

for $\varphi \in C_{0}^{\infty}(\Omega)$. Let us define $H_{0}(\Omega)$ to be the completion of $C_{0}^{\infty}(\Omega)$ in this norm. Let us set

$$
H(\Omega, \mathrm{loc})=\left\{\varphi \in L^{2}(\Omega, \mathrm{loc}): \chi \varphi \in H_{0}(\Omega) \text { for all } \chi \in C_{0}^{\infty}(\Omega)\right\} .
$$

The norm $\|\mid\|$ and the spaces $H_{0}$ and $H(\Omega$, loc $)$ are invariant under holomorphic mappings. The topology of $H(\Omega$, loc) seems to be useful for the study of plurisubharmonic functions because of the following properties.

THEOREM 7.5. If $\Omega \Subset \mathrm{C}^{n}$, then

(a) $L^{\infty}(\Omega$, loc $) \cap P(\Omega) \subset H(\Omega$, loc $)$;

(b) if $u_{j}, u \in L^{\infty}(\Omega, \operatorname{loc}) \cap P(\Omega)$, if $\lim _{j \rightarrow \infty} u_{j}=u$ almost everywhere on $\Omega$, and if $\left\{u_{j}\right\}$ is a montone sequence, then $u_{j}$ converges to $u$ in $H(\Omega, l o c)$;

(c) if, in addition, $\Omega$ is pseudoconvex, then $C^{\infty}(\Omega) \cap P(\Omega)$ is dense in $L^{\infty}(\Omega, \operatorname{loc}) \cap P(\Omega)$ in the topology of $H(\Omega$, loc $)$;

(d) the translation operator $\tau \mapsto u_{\tau}(z)=u(z-\tau)$ is continuous on $H(\Omega$, loc $)$ for each $u \in P(\Omega) \cap L^{\infty}(\Omega$, loc $)$.

The proof of Theorem 7.5 follows the arguments given in Section 3. That is, $u=\varphi+\psi$, where $\varphi \in C(\Omega)$, and $\psi \in L^{\infty}(\Omega, \operatorname{loc})$ with $C(\operatorname{supp} \psi)<\varepsilon$. Thus for instance

$$
\chi\left(u-u_{\tau}\right)=o(1)+\chi\left(\psi-\psi_{\tau}\right)
$$

and $C\left(\operatorname{supp}\left(\psi-\psi_{\tau}\right)\right) \leqslant 2 \varepsilon$.

\section{Capacitability}

In this section we show that the capacity $C^{*}$ in fact satisfies the axioms (8.1), (8.2) and (8.3) below and thus is a capacity in the generalized sense (see Choquet [12] and Brelot [7]). Some conclusions are drawn from this.

PROPOSITION 8.1. If $E_{1} \subset E_{2} \subset \ldots \subset \Omega$, and if $E=\cup E_{j}$ then $u_{E}^{*}=\lim _{j \rightarrow \infty} u_{E_{j}}^{*}$.

Proof. By Theorem 7.1, $u_{E_{j}}^{*=-1}$ on $E_{j}$ except on a pluripolar set. Thus, $\lim _{j \rightarrow \infty} u_{E_{j}}^{*}=-1$ on $E$, except on a pluripolar set. The result follows. 
THEOREM 8.2. Let $\Omega \Subset \mathrm{C}^{n}$ be strongly pseudoconvex. The set function $E \mapsto C^{*}(E)$ $=C^{*}(E, \Omega)$ satisfies:

$$
C^{*}\left(E_{1}\right) \leqslant C^{*}\left(E_{2}\right) \text { for } E_{1} \subset E_{2} \subset \Omega \text {; }
$$

if $K_{1} \supset K_{2} \supset$... are compact sets in $\Omega$ and $K=\cap K_{j}$, then $C^{*}(K)=\lim _{j \rightarrow \infty} C^{*}\left(K_{j}\right)$;

$$
\text { if } E_{1} \subset E_{2} \subset \ldots \subset \Omega \text { and } E=\cup E_{j} \text {, then } C^{*}(E)=\lim _{j \rightarrow \infty} C^{*}\left(E_{j}\right) \text {. }
$$

Proof. Assertion (8.1) follows from Proposition 6.1; (8.2) from Proposition 6.4. To prove (8.3), we only have to show $C^{*}(E) \leqslant \lim _{j \rightarrow \infty} C^{*}\left(E_{j}\right)$. It is no loss of generality to suppose that $E_{j} \Subset \Omega$. Let $u_{j}=u_{E_{j}}^{*}$, and let $\varepsilon, \eta>0$. Let $G$ be an open subset of $\Omega$ with $C^{*}(G)<\varepsilon$ and $G \supset \cup_{j}\left\{z \in E_{j}: u_{j}(z)>-1\right\}$. Set $u_{j}=\left\{u_{j}<-1+\eta\right\}$, and $\mathcal{O}_{j}=G \cup u_{j}$. Then, by Corollary 4.3,

$$
C\left(\mathscr{O}_{j}\right) \leqslant \varepsilon+\int\left(d d^{c} u_{\mathfrak{u}_{j}}^{*}\right)^{n}<\varepsilon+(1-\eta)^{-n} \int\left(d d^{c} u_{j}\right)^{n}=\varepsilon+(1-\eta)^{-n} C^{*}\left(E_{j}\right)
$$

Further, $E_{j} \subset \mathcal{O}_{j}$ and $\mathscr{O}_{1} \subset \mathscr{O}_{2} \subset \ldots$, since $u_{1} \geqslant u_{2} \geqslant \ldots$ Thus, $\mathscr{O}=\cup \mathcal{O}_{j} \supset E$ and

$$
C^{*}(\mathscr{O})=\lim _{j \rightarrow \infty} C\left(\mathcal{O}_{j}\right) \leqslant \varepsilon+\lim _{j \rightarrow \infty} C^{*}\left(E_{j}\right)(1-\eta)^{-n} .
$$

Letting $\varepsilon, \eta \rightarrow 0$ yields the assertion.

We recall the terminology that a set $E \subset \Omega$ is $\mathscr{K}$-analytic if it may be obtained from a Souslin operation on the compact subsets of $\Omega$. In particular, the Borel subsets of $\Omega$ are $\mathscr{K}$-analytic. The famous capacitability result of Choquet [12] is the following.

THEOREM (Choquet). If $C^{*}$ is a set function satisfying (8.1)-(8.3), then for every $\mathscr{H}$-analytic set $\mathrm{E} \subset \Omega$

$$
C^{*}(E)=\sup \{C(K): K \subset E \text { is compact }\} .
$$

An immediate consequence of this theorem and Theorem 6.9 is:

THEOREM 8.3. If $E \subset \Omega$ is a $\mathscr{K}$-analytic set, then $E$ is pluripolar if and only if every compact subset of $E$ is pluripolar.

In the case $\Omega=\mathrm{C}^{n}$, another approach to the capacitary extremal function has been given by Zaharjuta [28] and Siciak [26]. For $E \subset C^{n}$, we may set $V_{E}(z)=\sup \left\{v(z): v \in P\left(\mathbf{C}^{n}\right), \quad v<0\right.$ on $E$, and $v(\zeta)-\log (1+|\zeta|)$ is bounded above $\}$. (8.4) 
It follows (see Siciak [25]) that if $V_{E}^{*}$ is bounded above on an open set, then $V_{E}(\xi)-$ $\log (1+|\xi|)$ is bounded above on $\mathbf{C}^{n}$.

Now let $\mu$ be a finite nonnegative Borel measure on $\Omega$ such that $\mu(S)=0$ for every pluripolar set $S$. For instance, by Theorem 7.4, we may take $\mu=\left(d d^{c} u_{E}^{*}\right)^{n}$ for $E \subset \Omega$.

PROPOSITION 8.4. The set functions

$$
\gamma(E)=\int\left|u_{E}^{*}\right| d \mu
$$

and

$$
\gamma^{\prime}(E)= \begin{cases}\exp \left[-V_{E}^{*} d \mu\right] & \text { if } V_{E}^{*}<+\infty \\ 0 & \text { if } V_{E}^{*}<+\infty\end{cases}
$$

satisfy (8.1), (8.2), and (8.3).

Proof. First we consider the function $u_{E}^{*}$. Assertion (8.1) follows by Proposition 6.2. Similarly, (8.2) follows from Proposition 6.4 and Theorem 7.1, while (8.3) follows from Proposition 8.1. The arguments for $V_{E}^{*}$ are similar, the main difference being that in the proof of Proposition 6.4, we note that the convolutions $\chi_{\varepsilon} * \log (1+|z|)$ decrease uniformly to $\log (1+|z|)$ on $\mathbf{C}^{n}$.

Remark. Capacities like $\gamma, \gamma^{\prime}$ have been considered by Cegrell [10] and Sadullaev [25].

THEOREM 8.5. Let $\Omega$ be a bounded open set. If $E \subset \Omega$ is a $\mathscr{K}$-analytic set, then there is an $F_{\sigma}$-set $F$ and $a G_{\delta}$-set $G$ such that $F \subset E \subset G$ and $u_{F}^{*}=u_{E}^{*}=u_{G}^{*}, V_{E}^{*}=V_{F}^{*}=V_{G}^{*}$.

Proof. We may take $\mu$ to be Lebesque measure on $\Omega$. By Choquet's theorem and Proposition 8.4, it follows that $E$ is $\gamma$-capacitable; i.e.

$$
\gamma(E)=\sup \{\gamma(K): K \subset E, K \text { compact }\} .
$$

Thus, we have $F=\bigcup K_{j} \subset E$ such that $\int\left|u_{E}^{*}\right| d \mu=\int\left|u_{F}^{*}\right| d \mu$. Since $\left|u_{E}^{*}\right| \geqslant\left|u_{F}^{*}\right|$, it follows that $u_{E}^{*}=u_{F}^{*}$ almost everywhere and thus $u_{E}^{*}=u_{F}^{*}$. The existence of $G$ is elementary (c.f. the remark following Proposition 6.5). Similar arguments give the result for $V_{E}^{*}$.

\section{Balayage}

The classical ideas of "balayage" and "reduction" of a superharmonic function $u$ can be viewed either as solving a Dirichlet problem

$$
\Delta \tilde{u}=0 \text { on } \omega \Subset \Omega, \quad \tilde{u}=u \text { on } \partial \omega
$$


or as computing an envelope

$$
\vec{u}=\inf \{v: v \text { superharmonic on } \Omega, v \geqslant u \text { on } \partial \omega\} .
$$

Here we consider the analogous problems for plurisubharmonic functions. We will show that envelopes like (2) are characterized by a Dirichlet problem analogous to (1), but with the Laplacian replaced by $\left(d d^{c}\right)^{n}$. The basic quantitative result is an $L^{p}$ estimate for the distance from a function to its associated envelope (Corollary 9.8).

Proposition 9.1. Let $\Omega$ be open in $\mathbf{C}^{n}$ and $\psi \in P(\Omega) \cap L^{\infty}(\Omega$, loc $)$. If $D \Subset \Omega$ is strongly pseudoconvex, then there exists a unique function $\tilde{\psi} \in P(\Omega) \cap L^{\infty}(\Omega, \operatorname{loc})$ such that

$$
\begin{gathered}
\left(d d^{\mathrm{c}} \tilde{\psi}\right)^{n}=0 \text { on } D, \\
\tilde{\psi}=\psi \text { on } \Omega \backslash D .
\end{gathered}
$$

Further, $\tilde{\psi} \geqslant \psi$.

Proof. Let $\psi_{j}$ be continuous plurisubharmonic functions which decreases to $\psi$ on a neighborhood of $\bar{D}$. By Theorem 8.3, p. 42 of [4], there exists $\bar{\psi}_{j} \geqslant \psi_{j}$ continuous and plurisubharmonic on a neighborhood of $\bar{D}$ satisfying (9.1) and (9.2) with $\psi$ replaced by $\psi_{j}$. Then $\tilde{\psi}=\lim _{j} \tilde{\psi}_{j}$ satisfies (9.1) by Theorem 2.1. Since $\psi_{j} \downarrow \psi$ on a neighborhood of $\bar{D}$ and $\tilde{\psi}=\psi_{j}$ on $\Omega \backslash D$, the function $\tilde{\psi}=\psi$ on $\Omega \backslash D$ satisfies (9.2) also. Further, $\tilde{\psi} \geqslant \psi$ since $\tilde{\psi}=\psi_{j}$. The uniqueness follows from the domination theorem, Corollary 4.5 .

Now we consider some envelope functions more general than $u_{K}^{*}$.

COROLlARY 9.2. Let $h$ be a bounded, lower semicontinuous function on $\bar{\Omega} \Subset \mathrm{C}^{n}$. Then if

$$
u_{h}(z)=\sup \left\{v(z): v \in P(\Omega) \cap L^{\infty}(\Omega, \operatorname{loc}), v \leqslant h\right\}
$$

and

$$
u_{h}^{*}(z)=\limsup _{\zeta \rightarrow z} u_{h}(\zeta) \in P(\Omega) \cap L^{\infty}(\Omega)
$$

then

$$
\left(d d^{c} u_{h}^{*}\right)^{n}=0 \text { on the open set }\left\{u_{h}^{*}<h\right\} .
$$

Proof. If $z \in\left\{u_{h}^{*}<h\right\}$, then because $u_{h}^{*}$ is upper semicontinuous and $h$ is lower semicontinuous there exists a small ball $D$ centered at $z$ such that $\sup \{u(\zeta): \zeta \in \bar{D}\}<$ 
$\inf \{h(\zeta): \zeta \in \bar{D}\}$. If $\tilde{\psi}$ is the function given by Proposition 9.1. with $\psi=u_{h}^{*}$, we therefore have that $\tilde{\psi} \leqslant h$. Hence $\tilde{\psi} \leqslant u_{h}^{*}$, so $\tilde{\psi} \leqslant u_{h}^{*}$. Since $\left(d d^{c} \tilde{\psi}\right)^{n}=0$ on $D$, we therefore have that $\left(d d^{c} u_{h}^{*}\right)^{n}=0$ on $D$. Because $D$ is a neighborhood of an arbitrary point $z \in\left\{u_{h}^{*}<h\right\}$, the corollary is proved.

Let $F \subset \Omega \subset C^{n}$, be a closed subset of $\Omega$ such $\Omega \backslash F \Subset \Omega$. If $\psi \in P(\Omega) \cap L^{\infty}(\Omega$, loc $)$, then

and

$$
\psi_{F}(z)=\sup \{v(z): v \in P(\Omega), \quad v \leqslant \psi \text { on } F\}
$$

$$
\psi_{F}^{*}(z)=\limsup _{\zeta \rightarrow z} \psi(\zeta)
$$

COROLlaRY 9.3. If $\psi_{F}^{*}$ is as above, then

$$
\left(d d^{\mathcal{c}} \psi_{F}^{*}\right)^{n}=0 \text { on } \Omega \backslash F .
$$

Proof. If $D$ is a small ball in $\Omega \backslash F$, then $\tilde{\psi}_{F}^{*}=\psi_{F}^{*}$, exactly as in the previous corollary. Hence, $\left(d d^{c} \psi_{F}^{*}\right)^{n}=0$ on $D$, therefore on $\Omega \backslash F$.

Generally, Proposition 9.1 implies that "free upper envelopes" of plurisubharmonic functions satisfy the complex Monge-Ampère equation $\left(d d^{c} u\right)^{n}=0$. Corollaries 9.2 and 9.3 are examples. For another example, recall the function $V_{E}^{*}$ defined in (8.4).

COROLlary 9.4. $\left(d d^{\mathfrak{c}} V_{E}^{*}\right)^{n}=0$ on $\mathrm{C}^{n} \backslash \bar{E}$.

Proof. Same as the previous corollaries.

We note that the function $\psi_{F}^{*}$ can also be obtained by the method of balayage. Namely, let $\mathcal{U}$ be an open cover of $\Omega \backslash F$ by relatively compact balls $B_{1}, B_{2}, \ldots$, each of which is repeated infinitely often in the sequence. Let $\psi_{0}, \psi_{1}, \psi_{2}, \ldots$ be the sequence of plurisubharmonic functions on $\Omega$ obtained by putting $\psi_{0}=\psi$ and $\psi_{j+1}=\tilde{\psi}_{j}$, where $\tilde{\psi}_{j}$ is obtained from $\psi_{j}$ by Proposition 9.1 with $D=B_{j}$. Clearly, $\psi_{1} \leqslant \psi_{2} \leqslant \ldots, \psi_{j}=\psi$ on $F$ so the "balayage" of $\psi$, defined by

$$
\mathscr{B}(\psi, F)=\left(\sup \psi_{j}\right)^{*}
$$

satisfies

$$
\mathscr{B}(\psi, F) \leqslant \psi_{F}^{*} .
$$

Also, because each ball occurs infinitely many times in the sequence, it follows that

$$
\left[d d^{c} \mathscr{B}(\psi, F)\right]^{n}=0 \text { on } \Omega \backslash F
$$

by Theorem 7.4 (or Proposition 5.2). 
PROPOSITION 9.5. $\mathscr{B}(\psi, F)=\psi_{F}^{*}$ if $\Omega \backslash F \Subset \Omega$.

Proof. This follows from (9.4) and (9.5) and the domination principle, Corollary 4.5, provided we can show that $\left\{\mathscr{B}(\psi, F)<\psi_{F}^{*}\right\} \subset[\Omega \backslash F] \cup E$, where $E$ is a pluripolar set. But, clearly, from the definition of $\mathscr{B}(\psi, F)$ and $\psi_{F}^{*}, \mathscr{B}(\psi, F)=\psi *$ * $=\psi$, except on a pluripolar set in $\Omega \backslash F$, so the proposition follows.

Now let $F^{\prime}, F^{\prime \prime}$ be closed subsets of $\Omega$ such that $\Omega \backslash\left(F^{\prime} \cap F^{\prime \prime}\right) \Subset \Omega$. We may consider a sequence of "alternating" envelopes. Starting with $\psi \in P(\Omega) \cap L^{\infty}(\Omega, \operatorname{loc})$ we may take $\psi^{1}=\psi_{F}^{*}, \psi^{2}=\left(\psi_{F^{\prime \prime}}^{1}\right)^{*}, \psi^{3}=\left(\psi_{F^{\prime}}^{2}\right)^{*}, \ldots$, etc. If we define $T \psi=\left(\left(\psi_{F^{\prime}}\right)_{F^{\prime \prime}}^{*}\right)^{*}$, then from Proposition 9.5 we see that repeated alternating balayage, gives balayage from the intersection; i.e.

$$
\left(\lim _{j \rightarrow \infty} T^{j} \psi\right)^{*}=\psi_{F^{\prime} \cap F^{\prime \prime}}^{*}
$$

PROPOSITION 9.6. Let $\Omega$ be a strongly pseudoconvex set in $\mathrm{C}^{n}$, and $u, v \in P(\Omega) \cap L^{\infty}(\Omega)$ such that $\liminf _{\zeta \rightarrow \Omega \Omega} u(\zeta)-v(\zeta) \geqslant 0$. Then for all $t>0$,

$$
C\{v \geqslant u+t\} \leqslant \frac{1}{t^{n}}\left[\int_{\Omega}\left(d d^{\mathrm{c}} u\right)^{n}-\int_{\{v>u+t\}}\left(d d^{\mathrm{c}} v\right)^{n}\right]
$$

Proof. Replacing $v$ by $t v, u$ by $t u$, we see it is no loss of generality to take $t=1$. Set $E=\{v \geqslant u+1\}$ and let $K$ be a compact subset of $E$. Then if $F=\left\{u_{K}^{*}+v \geqslant u\right\}$ we have that $F \supset K \backslash\left\{u_{K}^{*}>-1\right\}$. Thus, $F \supset K$, except for a pluripolar set. By the comparison Theorem 4.2 , then,

$$
\int_{F}\left[d d^{\mathfrak{c}}\left(u_{K}^{*}+v\right)\right]^{n} \leqslant \int_{F}\left(d d^{c} u\right)^{n}
$$

But,

$$
\int_{F}\left[d d^{\mathfrak{c}}\left(u_{K}^{*}+v\right)\right]^{n} \geqslant \int_{K}\left(d d^{\mathfrak{c}} u_{K}^{*}\right)^{n}+\int_{F}\left(d d^{\mathfrak{c}} v\right)^{n}=C(K)+\int_{F}\left(d d^{\mathfrak{c}} v\right)^{n}
$$

Consequently,

$$
C(K) \leqslant \int_{F}\left(d d^{c} u\right)^{n}-\left(d d^{c} v\right)^{n}
$$

Let $K_{1} \subset K_{2} \subset \ldots$ be a sequence of compact subsets of $E$ such that $C\left(K_{j}\right) \rightarrow C(E)\left(=C^{*}(E)\right.$ since $E$ is a Borel set). Then $u_{K_{1}}^{*} \geqslant u_{K_{2}}^{*} \geqslant \ldots$ and, $\inf u_{K_{j}}^{*}=w \in P(\Omega)$. The sets $F_{j}=$ $\left\{u_{K_{j}}^{*}+v \geqslant u\right\}$ therefore decrease to $\{w+v \geqslant u\}$. So, from (9.6) we have 


$$
C(E) \leqslant \int_{\{w+v \geqslant u\}}\left(d d^{\mathfrak{c}} u\right)^{n}-\left(d d^{\mathfrak{c}} v\right)^{n}
$$

However, $\{v \geqslant u+1\} \subset\{w+v \geqslant u\} \subset \Omega$, so the Proposition follows.

We will estimate the distance from $\psi$ to $\psi_{F}$ in terms of the Choquet integral, which may be defined as

$$
\int_{E}|f| d C=\int_{0}^{+\infty} C(\{|f|>t\} \cap E, \Omega) d t
$$

for any Borel measurable function $f$ and any Borel subset $E \subset \Omega$.

THEOREM 9.7. Let $\Omega \Subset \mathbf{C}^{n}$ be strongly pseudoconvex. Let $F$ be closed in $\Omega$ with $\Omega \backslash F \Subset \Omega$. Then for $1 \leqslant \alpha<n$ and for $\psi \in P(\Omega) \cap L^{\infty}(\Omega$, loc $)$

$$
\int_{\Omega}\left(\psi_{F}^{*}-\psi\right)^{\alpha} d C \leqslant \frac{C(\Omega \backslash F, \Omega)^{1-\alpha / n}}{1-\alpha / n}\left(\int_{\Omega}\left(d d^{\mathrm{c}} \psi\right)^{n}\right)^{\alpha / n}
$$

Proof. In order to estimate the Choquet integral, we note that

$$
\begin{aligned}
\left.C\left\{\psi_{F}^{*}-\psi\right)^{\alpha}>t\right\} & \leqslant C\left(\left\{\psi_{F}^{*} \geqslant \psi+t^{1 / \alpha}\right\} \cap \Omega \backslash F\right) \\
& \left.\leqslant \min \left\{C(\Omega \backslash F), t^{-n / \alpha} \int_{\Omega} d d^{c} \psi\right)^{n}\right\}
\end{aligned}
$$

where the last inequality is obtained from Proposition 9.6. But

$$
\int_{0}^{+\infty} \min \left(a, t^{-\beta} b\right) d t=\frac{\alpha \beta}{\beta-1}\left(\frac{b}{a}\right)^{1 / \beta}(\beta>1)
$$

from which the estimate follows by direct substitution.

We note that these estimates apply also to the solution of the Dirichlet problem

$$
v \in P(\Omega) \cap C(\bar{\Omega}), \quad\left(d d^{c} v\right)^{n}=0 \text { on } \Omega, \quad v=\varphi \text { on } \Omega .
$$

for $\Omega \Subset \mathbf{C}^{n}$ strongly pseudoconvex and smoothly bounded, and $\varphi \in C(\partial \Omega)$. If $u_{\varphi}$ is the function defined in Corollary 9.2, then by Theorem 8.3 of [4], $u_{\varphi}$ is the solution of (9.8). By the preceding discussion it is clear that $u_{\varphi}$ is also equal to the balayage

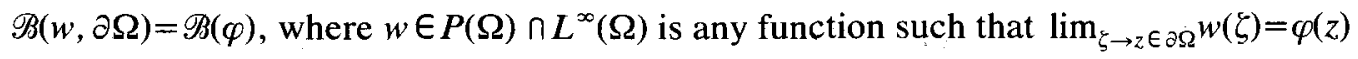
for all $z \in \partial \Omega$. This is like Proposition 9.5 except that boundary continuity is used to replace the condition $\Omega \backslash F \Subset \Omega$. 
COROLLARY 9.8 Let $\Omega \Subset \mathrm{C}^{n}$ be strongly pseudoconvex. Let $u \in P(\Omega) \cap L^{\infty}(\Omega)$, $\varphi \in C(\partial \Omega)$ satisfy $\lim _{\zeta \rightarrow z} \in \partial \Omega(\zeta)=\varphi(z)$ for all $z \in \partial \Omega$. Then the solution of (9.8), i.e., $u_{\varphi}=\mathscr{B}(u)$, satisfies

$$
\int_{K}(\mathscr{B} u-u)^{\alpha} d C \leqslant \frac{C(K)^{1-\alpha / n}}{1-\alpha / n}\left(\int_{\Omega}\left(d d^{\mathcal{c}} u\right)^{n}\right)^{\alpha / n}
$$

for $1 \leqslant \alpha<n$ and $K \subset \Omega$ compact.

Proof. This is essentially the same as the previous proof. This time we use Proposition 9.6 to obtain

$$
C\left(\left\{(\mathscr{B} u-u)^{\alpha}>t\right\} \cap K\right) \leqslant \min \left\{C(K), t^{-n / \alpha} \int_{\Omega}\left(d d^{c} u\right)^{n}\right\}
$$

from which the estimate follows.

For a Borel measure $\mu$ on $\Omega$, we will write $\mu \ll C$ if $\mu(E) \leqslant C(E, \Omega)$ for all Borel sets $E$ $\Omega$. Clearly, any measure of the form $\mu=\left(d d^{\mathrm{c}} v\right)^{n}$ with $v \in P(\Omega), 0<v<1$, satisfies $\mu \ll C$. For instance, if $v=$ constant $|z|^{2}$ then $\left(d d^{c} v\right)^{n}$ is a constant multiple of Lebesgue measure.

COROLlaRy 9.9. For $u$ and $\Omega$ as in Corollary 9.8 and for $\mu \ll C, 1 \leqslant \alpha<n$,

$$
\int(\mathscr{B} u-u)^{\alpha} d \mu \leqslant \frac{\mu(\Omega)^{1-\alpha / n}}{1-\alpha / n}\left(\int_{\Omega}\left(d d^{\mathrm{c}} u\right)^{n}\right)^{\alpha / n} .
$$

Proof. We estimate

$$
\begin{aligned}
\mu\left\{(\mathscr{B} u-u)^{\alpha}>t\right\} & \leqslant \min \left\{\mu(\Omega), C\left\{(\mathscr{B} u-u)^{\alpha}>t\right\}\right. \\
& \leqslant \min \left\{\mu(\Omega), t^{-n / \alpha} \int_{\Omega}\left(d d^{c} u\right)^{n}\right\}
\end{aligned}
$$

and proceed as before to complete the proof.

Finally, we remark that if $\mu \ll C$, then $\mu(S)=0$ for negligible sets $S$. Thus we may consider the capacity $\gamma$ defined by (8.5). If $1 \leqslant \alpha<n$, then for any Borel set $E \Subset \Omega$, Corollary 9.9 gives

$$
\gamma(E, \Omega)=\int\left|u_{E}^{*}\right| d \mu \leqslant\left(\int_{\Omega} \mid u_{E}^{* \mid \alpha} d \mu\right)^{1 / \alpha} \leqslant \frac{\mu(\Omega)^{1-\alpha / n}}{1-\alpha / n}\left(\int_{\Omega}\left(d d^{c} u_{E}^{*}\right)^{n}\right)^{\alpha / n}
$$

We conclude that if $\mu(\Omega)<\infty$ then $C$ dominates the capacity $\gamma$ in this sense. 


\section{Thinness}

Now we apply our earlier results to thin sets. First, we recall the definition of thinness (see e.g. [7]), translated to the plurisubharmonic case. A set $S \subset \mathbf{C}^{n}$ is thin at a point $z$ if $z \in \bar{S}$ and there is an open set $\mathscr{U} \ni z$ and a function $\psi \in P(\mathscr{U})$ such that

$$
\limsup _{\substack{\zeta \rightarrow z \\ \zeta \in S}} \psi(\zeta)<\psi(z)
$$

We let the "effilé" part of $S$ be denoted by

$$
e(S)=\{z \in \bar{S}: S \text { is thin at } z\} .
$$

The situation here is different from the subharmonic case where polar sets are always thin.

Example. If $S=\left\{z \in \mathbf{C}^{2}:\left|z_{1}\right|<1, z_{2}=0\right\}$, then $S$ is pluripolar but $S$ is nowhere thin.

For $S \subset \Omega \Subset \mathrm{C}^{n}$ we denote the set where $S$ is negligible by

$$
N(S)=N=\left\{z \in \bar{S} ; u_{S}^{*}>-1\right\} .
$$

And, if $\left\{\mathcal{O}_{j}\right\}$ is a neighborhood base for $\Omega$, we set

$$
\tilde{N}(S)=\tilde{N}=\bigcup_{j} N\left(O_{j} \cap S\right)
$$

The definition of $\tilde{N}$ is independent of the choice of $\left\{\mathcal{O}_{j}\right\}$, and $\tilde{N}(S) \supset N(S)$. But, by the example we may have $e(S)=\varnothing$ and $N(S)=\bar{S}$.

For general $S$, the set $N$ may have positive Lebesgue measure. However, it lies "outside" of $S$ in the following sense.

PROPOSITION 10.1. If E is a $\mathscr{K}$-analytic set, then

$$
\int_{N}\left(d d^{c} u_{E}^{*}\right)^{n}=0
$$

Proof. By Theorem 8,5 there exist $K_{1} \subset K_{2} \subset \ldots \subset E$ such that $\lim _{j \rightarrow \infty} u_{K_{j}}^{*}=u_{E}^{*}$. It follows from Proposition 5.3 and Theorem 2.1 that

$$
\int\left(d d^{c} u_{E}^{*}\right)^{n}=\lim _{j \rightarrow \infty} \int\left(d d^{c} u_{K_{j}}^{*}\right)^{n}=\lim _{j \rightarrow \infty}-\int u_{K_{j}}^{*}\left(d d^{c} u_{K_{j}}^{*}\right)^{n} .
$$


By Theorem 2.4, we have

$$
\int\left(d d^{c} u_{E}^{*}\right)^{n}=-\int u_{E}^{*}\left(d d^{c} u_{E}^{*}\right)^{n}
$$

which completes the proof, since $-1 \leqslant u_{E}$.

We may also use the characterization of thinness in Brelot [7].

PROPOSITION 10.2. If $S$ is thin at $z$, then there exists a neighborhood $\mathscr{\ddots} z_{z_{0}}$ and $a$ function $\varphi \in P(\mathcal{U})$ such that $\varphi(z)>-\infty$ and

$$
\limsup _{\substack{\zeta \rightarrow z \\ \zeta \in S}} \varphi(\zeta)=-\infty
$$

Proof. By hypothesis, there exists an open $U$ and $\psi \in P(\mathcal{U})$ such that $\lim \sup _{\zeta \rightarrow z} \psi(\zeta)<\psi(z)$. Without loss of generality, we may assume that $z=0$, $u=\{|z|<1\}, \psi<0$ on $\mathcal{U}, \psi<-1$ on $\mathcal{U} \cap S$, and $\psi(0)=-\eta>-1$. By the upper semicontinuity of $\psi$, we may choose $\delta_{j}>0$ (for $j$ sufficiently large) such that $\psi(z)<-\eta+2^{-j-1}$ for $|z|<\delta_{j}$. It follows that the function

$$
\varphi_{j}=\left\{\begin{array}{l}
\max \left(\frac{2^{-j-1}}{\left|\log \delta_{j}\right|} \log |z|, \psi+2^{-j}\right), \quad|z|<\delta_{j} \\
\frac{2^{-j-1}}{\left|\log \delta_{j}\right|} \log |z|, \quad \delta_{j} \leqslant|z|<1
\end{array}\right.
$$

is plurisubharmonic on $\mathcal{U}$. Further, $\varphi_{j}(0)=-2^{-j}$ and $\varphi_{j}(\zeta) \leqslant-1+\eta-2^{-j}$ for $\zeta \in S$, $\zeta$ sufficiently close to 0 . Thus $\varphi=\Sigma \varphi_{j}$ is the desired function.

PROPOSITION 10.3. If $S$ is thin at $z$, then there exists a neighborhood $\mathcal{U}$ of $z$ such that $u_{S^{\prime}}(z)>-1$ where $S^{\prime}=(\mathcal{U} \cap S) \backslash\{z\}$. In particular, the set of thin points is contained in $N(S)$, i.e., $\tilde{N}(S) \supset N(S) \supset e(S)$.

Proof. We let $\varphi$ be the function given by Proposition 9.14. Then we may choose a neighborhood $U^{\prime}$ of $z$ such that $\varphi(\xi)<\varphi(z)-1$ for $z \neq \xi \in \mathcal{U}^{\prime} \cap S$. It follows that

$$
u_{s \cap u^{\prime}}(z)>\frac{\varphi(z)}{C}>-1
$$

where $C=\sup \left\{\varphi(\zeta): \xi \in U^{\prime} \cap S\right\}$

The following result and its proof are essentially taken from Choquet [14]. By the previous proposition, this gives an estimate on the size of the thin points. 
THEOREM 10.4. If $S \subset \Omega$ is an arbitrary subset, then for $\varepsilon>0$ there exists an open set $U$ containing $\tilde{N}(S)$ such that $C^{*}(\mathcal{U} \cap S)<\varepsilon$.

Proof. Let $\left\{\mathscr{O}_{j}\right\}$ denote a countable base for the topology of $\mathbf{C}^{n}$. Let

$$
S_{j}=\left\{u_{C_{j} \cap S}^{*}>-1\right\} \cap \bar{S} .
$$

Now by Theorem 7.1, the set $S \cap\left(\cup S_{j}\right)$ is pluripolar, so we can find an open set $\mathscr{O}_{0}$ containing it and such that $C^{*}\left(\mathscr{O}_{0}\right)<\varepsilon / 2$. By Theorem 3.5, there exist open sets $\omega_{j}$ with $C^{*}\left(\omega_{j}\right)<\varepsilon 2^{-j-1}$ and such that $u{ }_{S}^{*} \cap \mathscr{O}_{j}$ is continuous on $\Omega \backslash \mathscr{O}_{j}$.

We set

$$
T=S \cap\left[\bigcap_{j=0}^{\infty} \Omega \backslash \mathcal{O}_{j}\right]
$$

Thus $\bar{T} \cap \mathcal{O}_{j}=\varnothing$ for all $j$. By the choice of $\mathscr{O}_{0}$, it follows that $u_{\mathcal{C}_{j} \cap S}^{*}=-1$ on $T$, and by continuity, this holds on $\bar{T}$. Thus $\omega={ }^{\mathrm{c}} T$ is an open set, and $\tilde{N}(S) \subset \omega$. Further, we have $\omega \cap S \subset \cup_{j=0}^{\infty} \mathcal{O}_{j}$, and thus $C^{*}(\omega \cap S)<\varepsilon$.

As a corollary to Theorem 10.4, we have

COROLlaRY 10.5. If $S \subset \mathbf{C}^{n}$ then $S \cap \tilde{N}(S)$ is pluripolar. In particular, the set $e(S) \cap S$ of points of $S$ where $S$ is thin is pluripolar.

Finally we apply this to stability for balayage. Let $F \subset \Omega$ be a closed subset. A point $z \in F$ is stable if $\mathscr{B}(\psi, F \cup \partial \Omega, z)=\psi(z)$ for all $\psi \in C(\bar{\Omega}) \cap P(\Omega)$. An easy consequence of the definition of stability is the following:

If $z$ is stable then for each $\varphi \in C(\Omega)$, the envelope function

$$
u(F, \varphi, z)=\sup \{v(z): v \in P(\Omega), v(\zeta) \leqslant \varphi(\zeta) \text { for } \zeta \in F\}
$$

satisfies $u(F, \varphi, z)^{*} \leqslant \varphi(z)$.

By Theorem 2 of [3] the set of unstable points of $F$ is pluripolar, which generalizes the classical theorem of Kellogg and Evans for subharmonic functions. This result may also be obtained as a consequence of Theorem 10.4 because of the following.

PROPOSITION 10.6. The set of unstable points of a closed set $F \subset \Omega$ is the same as $\tilde{N}(F)$.

We omit the simple proof. 


\section{References}

[1] Alexander, H., Projective capacity. In Recent Developments in Several Complex Variables, pp. 3-27. J. E. Fornaess, ed., Princeton Univ. Press, 1981.

[2] BEDFORD, E., Extremal plurisubharmonic functions and pluripolar sets in $\mathbf{C}^{2}$. Math. Ann., $249(1980), 205-223$.

[3] - Envelopes of continuous, plurisubharmonic functions. Math. Ann., 251 (1980), 175-183.

[4] BEDFORD, E. \& TAYLOR, B. A., The Dirichlet problem for the complex Monge-Ampère equation. Invent. Math., 37 (1976), 1-44.

[5] - Variational properties of the complex Monge-Ampère equation, I: Dirichlet Principle. Duke Math. J., 45 (1978), 375-403.

[6] - Variational properties of the complex Monge-Ampère equation, II: Intrinsic norms. Amer. J. Math., 101 (1979), 1131-1166.

[7] Brelot, M., Elements de la théorie classique du potentiel. Centre de Documentation, Paris, 1959.

[8] Cartan, H., Capacité extérieure et suites convergentes de potentiels. C.R. Acad. Sci. Paris, (1942), 944-946.

[9] - Théorie du potentiel Newtonien: énérgie, capacité, suites de potentiels. Bull. Soc. Math. France, 73 (1945), 74-106.

[10] Cegrell, U., Construction of capacities on $\mathbf{C}^{n}$. Preprint.

[11] Chern, S. S., Levine, H. I. \& NirenberG, L., Intrinsic norms on a complex manifold. Global Analysis (Papers in honor of K. Kodaira), pp. 119-139. Univ. of Tokyo Press, Tokyo, 1969.

[12] Choquet, G., Theory of capacities. Ann. Inst. Fourier, 5 (1953), 131-292.

[13] - Potentiels sur un ensemble de capacité nulle. Suites de potentiels. C.R. Acad. Sci. Paris, 244 (1957), 1707-1710.

[14] - Sur les points d'effilement d'un ensemble. Application a l'étude de la capacité. Ann. Inst. Fourier, 9 (1959), 91-101.

[15] Federer, H., Geometric Measure Theory. Springer-Verlag, New York, 1969.

[16] Gaveau, B., Méthodes de contrôle optimal en analyse complexe, I: Résolution d'équations de Monge-Ampère. J. Funct. Anal., 25 (1977), 391-411.

[17] JoSEFSON, B., On the equivalence between locally polar and globally polar sets for plurisubharmonic functions on $\mathrm{C}^{n}$. Ark. Mat., 16 (1978), 109-115.

[18] Lelong, P., Plurisubharmonic functions and positive differential forms. Gordon and Breach, New York, 1969.

[19] - Functionelles analytiques et fonctions entieres ( $n$ variables). Univ. of Montreal, Montreal (1968).

[20] - Fonctions plurisousharmoniques et fonctions analytiques de variables réeles. Ann. Inst. Fourier, 16 (1966), 269-318.

[21] - Intégration sur en ensemble analytique complexe. Bull. Soc. Math. France, 85 (1957), 239-262.

[22] Molzon, R., Shiffman, B. \& Sibony, N., Average growth estimates for hyperplane sections of entire analytic sets. Math. Ann., 257 (1981), 43-59.

[23] RoNkIN, L., Regularization of the supremum of a family of plurisubharmonic functions and its application to analytic functions of several variables, Math. USSR-Sb., 71 (113), (1966), 132-142.

[24] Sadullaev, A., The operator $\left(d d^{c} u\right)^{n}$ and condenser capacities (Russian). Dokl. Akad. Nauk SSSR, 1980, 44-47.

[25] - Deficient divisors in the Valiron sense. Math. USSR-Sb., 36 (1980), 535-547.

[26] SiCiak, J., Extremal plurisubharmonic functions in $\mathbf{C}^{n}$. Proc. First Finnish-Polish Summer School in Complex Analysis, 1977, 115-152. 
[27] SiU, Y. T., Extension of meromorphic maps. Ann. of Math., 102 (1975), 421-462.

[28] ZaharjuTA, V. P., Extremal plurisubharmonic functions, orthogonal polynomials, and the Bernstein-Walsh theorem for analytic functions of several complex variables. Ann. Polon. Math., 33 (1976), 137-148.

Received May 5, 1981 